\title{
Bayesian Inference of Burr Type VIII Distribution Based on Censored Samples
}

\author{
Navid Feroz \\ Department of Mathematics and Statistics, Allama Iqbal Open University, Islamabad, Pakistan \\ Correspondence should be addressed to Navid Feroz; navidferoz@hotmail.com
}

Received 9 January 2014; Accepted 4 April 2014; Published 13 May 2014

Academic Editor: Hans Engler

Copyright (C) 2014 Navid Feroz. This is an open access article distributed under the Creative Commons Attribution License, which permits unrestricted use, distribution, and reproduction in any medium, provided the original work is properly cited.

\begin{abstract}
This paper is concerned with estimation of the parameter of Burr type VIII distribution under a Bayesian framework using censored samples. The Bayes estimators and associated risks have been derived under the assumption of five priors and three loss functions. The comparison among the performance of different estimators has been made in terms of posterior risks. A simulation study has been conducted in order to assess and compare the performance of different estimators. The study proposes the use of inverse Levy prior based on quadratic loss function for Bayes estimation of the said parameter.
\end{abstract}

\section{Introduction}

Burr [1] introduced twelve different forms of the cumulative distribution functions of Burr distribution. Among these twelve distribution functions, Burr type $\mathrm{X}$ and Burr type XII have been discussed by most of the analysts, while the remaining density functions are still waiting for the attention of the researchers. Soliman [2] derived the Bayes estimates of the parameter of Burr type XII distribution relative to quadratic, LINEX, and generalized entropy loss functions. Based on Lindley's approximation, the comparisons were made between maximum likelihood and Bayes estimation. Monte Carlo simulation was also used. Joshi [3] obtained the Bayesian estimation of reliability of generalized Burr distribution with the help of a censored sample. The precautionary and squared error loss functions were used for estimation. The noninformative and beta prior distributions were assumed for posterior analysis. Asgharzadeh and Valiollahi [4] considered the uniformly minimum variance unbiased (UMVU) of Burr model under progressively type II censored samples; the Bayes and empirical Bayes estimates for the unknown parameter and the reliability function of the Burr model were derived. The Bayes and empirical Bayes estimates were obtained on the basis of absolute error and logarithmic loss functions. A numerical example and a Monte Carlo simulation study were used to illustrate the applicability of the results. Saleem and Aslam [5] analyzed the parameter of the Rayleigh distribution under censored data. AL-Hussaini and Hussein [6] presented the maximum likelihood and Bayes estimators of the parameters, survival function (SF), and hazard rate function (HRF) for the threeparameter exponentiated Burr type XII distribution under type II censored scheme. Soliman et al. [7] dealt with Bayesian inference and prediction problems of the Burr type XII distribution based on progressive first failure censored data. Squared error loss function was proposed for estimation. Gibbs sampling procedure was applied to draw Markov chain Monte Carlo (MCMC) samples. A simulation study was carried out to compare the proposed Bayes estimators with the maximum likelihood estimators. A real life data set was also used to illustrate the results derived. Singh [8] discussed the uniformly minimum variance unbiased estimate (UMVUE) of the probability density function (pdf) of the Burr distribution. The UMVUE of the cumulative distribution function (CDF), pth quantile, and rth moment of the Burr distribution were also obtained. Feroze and Aslam [9] considered the Bayesian analysis of Gumbel type II distribution under doubly censored samples using different priors (informative and noninformative) and loss functions (symmetric and asymmetric). Bayesian credible intervals along with posterior predictive distributions were also derived. 
One of the important distributions from Burr family of distributions is Burr type VIII distribution. This distribution can be used to model the lifetime data. Burr [1] has indicated that this distribution can be used as an alternative lifetime model in many cases. Its two-parametric case gives more flexibility as compared to exponential and Rayleigh distribution in order to model lifetime data. In this paper, the Bayesian inference of Burr type VIII distribution has been considered using different loss functions and priors on the basis of three censoring techniques.

The probability density function (pdf) of Burr type VIII distribution is

$$
f(x, \psi)=\psi\left(\frac{2}{\pi}\right)^{\psi} \frac{e^{x}}{1+e^{2 x}}\left\{\tan ^{-1}\left(e^{x}\right)\right\}^{\psi-1} ; \quad \psi>0, x>0 .
$$

The cumulative distribution function (CDF) of the distribution is

$$
F(x, \psi)=\left\{\left(\frac{2}{\pi}\right) \tan ^{-1}\left(e^{x}\right)\right\}^{\psi}
$$

\section{Prior Distributions}

The prior distribution is a key part of Bayesian analysis and represents the information about an uncertain parameter that is combined with the probability distribution of new data to derive the posterior distribution, which in turn is used for future inferences and decision making. Therefore, prior subject-matter knowledge about a parameter is a vital aspect of the inference process. The prior distributions can be categorized as informative and noninformative priors. As their names suggest, the informative priors are used when the prior information regarding the current experiment is available (may be in the form of prior beliefs), while when such prior information is not available the use of noninformative priors becomes inevitable. We have assumed both informative and noninformative priors for the Bayesian analysis of the said parameter.

Among noninformative priors the uniform prior proposed by Laplace [10] and Jeffreys prior suggested by Jeffreys [11] are the most famous priors. We have assumed these priors along with some informative priors to derive the posterior distributions.

The uniform prior is assumed to be

$$
p(\psi) \propto 1 ; \quad \psi>0 .
$$

Jeffreys prior is defined to be

$$
\begin{aligned}
& p(\psi) \propto \sqrt{|I(\underline{\psi})|}, \\
& \text { where } I(\underline{\psi})=-E\left[\frac{\partial^{2} \ln f(x, \psi)}{\partial \psi^{2}}\right]=\frac{1}{\psi^{2}} ; \\
& \text { therefore } p(\psi) \propto \sqrt{|(\underline{\psi})|}=\frac{1}{\psi} .
\end{aligned}
$$

The informative prior for the parameter $\psi$ is assumed to be gamma distribution as

$$
p(\psi) \propto \psi^{a-1} e^{-b \psi} \quad a>0, b>0, \psi>0 .
$$

The informative prior for the parameter $\psi$ is assumed to be exponential distribution as

$$
p(\psi) \propto e^{-c \psi}, \quad c>0, \psi>0 .
$$

The informative prior for the parameter $\psi$ is assumed to be inverse Levy distribution as

$$
p(\psi) \propto \psi^{-1 / 2} e^{-0.5 d \psi}, \quad d>0, \psi>0
$$

The informative priors presented in (5)-(7) have been used in order to make our analysis more versatile. As the Burr type VIII distribution can be used to model the lifetime data, prior information available in such form can effectively be used. It should be noted that the gamma, exponential, and inverse Levy distributions can be used to model the lifetime data. So, these distributions can be used as prior distribution for the analysis of Burr type VIII distribution under Bayesian framework.

\section{Bayesian Estimation under Left Censored Samples}

Censoring is a useful technique when the value of a measurement or observation is only partially known. That is, it is used when an observed value is outside the range of a measuring instrument or the measure outside a range is not desired. It has a great usage in life testing experiments where continuity of the experiment till the last failure is not feasible. Censoring has many types. However, we have considered left, singly type II, and doubly type II censored samples for Bayesian inference of the parameter of Burr type VIII distribution.

Let $X_{m+1}, \ldots, X_{n}$ be the last $n-m$ order statistics from a sample of size $n$ from Burr type VIII distribution. Then the likelihood function for the $X_{m+1}, \ldots, X_{n}$ observations is

$$
L(\psi \mid x) \propto\left\{F\left(x_{m+1}, \psi\right)\right\}^{m} \prod_{i=m+1}^{n} f\left(x_{i}, \psi\right),
$$

$$
\begin{aligned}
& L(\psi \mid x) \\
& \quad \propto \psi^{n-m} e^{-\psi\left[\sum_{i=m+1}^{n} \ln \left\{(2 / \pi) \tan ^{-1}\left(e^{x_{i}}\right)\right\}^{-1}+m \ln \left\{(2 / \pi) \tan ^{-1}\left(e^{x_{m+1}}\right)\right\}^{-1}\right]} .
\end{aligned}
$$

The details for the derivation of the likelihoods function can be seen from the appendix. Now the generalized expressions for posterior distributions, Bayesian estimators, posterior risks, posterior predictive distributions, posterior predictive intervals, and credible intervals have been presented in the following. The expressions under priors given in (3), (4), (5), (6), and (7) can be derived by putting $l_{1}=0, l_{2}=0, l_{3}=0$, $l_{4}=0 ; l_{1}=1, l_{2}=0, l_{3}=0, l_{4}=0 ; l_{1}=0, l_{2}=1, l_{3}=0$, $l_{4}=0 ; l_{1}=0, l_{2}=0, l_{3}=1, l_{4}=0 ;$ and $l_{1}=0, l_{2}=0, l_{3}=1$, $l_{4}=1$, respectively, in the generalized results. 
The generalized posterior distribution under left censored samples is

$$
\begin{aligned}
p(\psi \mid x)= & \frac{\left\{C_{1}(x)+l_{2} c+l_{3} b+0.5 d l_{4}\right\}^{n-m+1-l_{1}+l_{3}(a-1)-0.5 l_{4}}}{\Gamma\left(n-m+1-l_{1}+l_{3}(a-1)-0.5 l_{4}\right)} \\
& \times \psi^{n-m-l_{1}+l_{3}(a-1)-0.5 l_{4}} e^{-\psi\left\{C_{1}(x)+l_{2} c+l_{3} b+0.5 d l_{4}\right\}},
\end{aligned}
$$

where

$$
\begin{aligned}
C_{1}(x)= & \sum_{i=m+1}^{n} \ln \left\{\left(\frac{2}{\pi}\right) \tan ^{-1}\left(e^{x_{i}}\right)\right\}^{-1} \\
& +m \ln \left\{\left(\frac{2}{\pi}\right) \tan ^{-1}\left(e^{x_{m+1}}\right)\right\}^{-1} .
\end{aligned}
$$

The details for the derivation of the posterior distribution can be seen from the appendix.

3.1. Loss Functions. The decision theory suggests that in order to select the best estimator a loss function must be specified and used to estimate the risk associated with each of the possible estimates. Since there is no definite analytical process that allows us to identify the proper loss function to be used, most of the analysts use the squared error loss function which is symmetrical and associates equal importance to the losses due to overestimation and underestimation of equal magnitude and obtain the posterior mean as Bayesian estimate. But in situations where the loss is asymmetric, we have to employ some asymmetric loss functions. So, here we have assumed some symmetric and asymmetric loss functions for the analysis of the parameter under study. The detail of these loss functions is as follows.

LINEX Loss Function (LLF). LINEX (linear exponential) loss function has been defined by Klebanov [12]. The expression of the loss function is $L\left(\psi_{\mathrm{LLF}}, \psi\right)=b\left[e^{a\left(\psi_{\mathrm{LLF}}-\psi\right)}-a\left(\psi_{\mathrm{LLF}}-\psi\right)-1\right]$.

For $a=b=1$, we have $L\left(\psi_{\mathrm{LLF}}, \psi\right)=e^{\left(\psi_{\mathrm{LLF}}-\psi\right)}-\left(\psi_{\mathrm{LLF}}-\right.$ $\psi)-1$. Then the Bayes estimator based on this loss function is $\psi_{\mathrm{LLF}}=-\ln \left[E\left(e^{-\psi}\right)\right]$.

Precautionary Loss Function (PLF). Norström [13] introduced an asymmetric precautionary loss function (PLF) which can be presented as $L\left(\psi_{\mathrm{PLF}}, \psi\right)=\left(\psi_{\mathrm{PLF}}-\psi\right)^{2} / \psi_{\mathrm{PLF}}$, where $\psi_{\mathrm{PLF}}=$ $\left[E\left(\psi^{2}\right)\right]^{1 / 2}$ is the Bayes estimator under this loss function.

Quadratic Loss Function (QLF). The quadratic loss function can be defined as $L\left(\psi, \psi_{\mathrm{QLF}}\right)=\left(\left(\psi-\psi_{\mathrm{QLF}}\right) / \psi\right)^{2}$. The Bayes estimator under QLF is given as $\psi_{\mathrm{QLF}}=E\left(\psi^{-1}\right)\left\{E\left(\psi^{-2}\right)\right\}^{-1}$.

Bayes estimator and corresponding risk under using LINEX loss function are, respectively, presented as

$$
\psi_{\mathrm{LLF}}=-\left\{n-m+1-l_{1}+l_{3}(a-1)-0.5 l_{4}\right\}
$$

$$
\begin{aligned}
& \times \ln \left\{\frac{C_{1}(x)+l_{2} c+l_{3} b+0.5 d l_{4}}{C_{1}(x)+l_{2} c+l_{3} b+0.5 d l_{4}+1}\right\}, \\
\rho\left(\psi_{\mathrm{LLF}}\right)= & \frac{n-m+1-l_{1}+l_{3}(a-1)-0.5 l_{4}}{C_{1}(x)+l_{2} c+l_{3} b+0.5 d l_{4}} \\
& +\left\{n-m+1-l_{1}+l_{3}(a-1)-0.5 l_{4}\right\} \\
& \times \ln \left\{\frac{C_{1}(x)+l_{2} c+l_{3} b+0.5 d l_{4}}{C_{1}(x)+l_{2} c+l_{3} b+0.5 d l_{4}+1}\right\} .
\end{aligned}
$$

The details for the derivation of the Bayes estimates and posterior risks under LLF can be seen from the appendix.

Bayes estimator and corresponding risk under precautionary loss function are, respectively, presented as

$$
\begin{aligned}
\psi_{\mathrm{PLF}}=( & \left\{n-m+1-l_{1}+l_{3}(a-1)-0.5 l_{4}\right\} \\
& \left.\times\left\{n-m+2-l_{1}+l_{3}(a-1)-0.5 l_{4}\right\}\right)^{1 / 2} \\
\times & \left(C_{1}(x)+l_{2} c+l_{3} b+0.5 d l_{4}\right)^{-1}, \\
\rho\left(\psi_{\mathrm{PLF}}\right)=2[( & \left(\left\{n-m+1-l_{1}+l_{3}(a-1)-0.5 l_{4}\right\}\right. \\
& \left.\times\left\{n-m+2-l_{1}+l_{3}(a-1)-0.5 l_{4}\right\}\right)^{1 / 2} \\
& \left.\times\left(C_{1}(x)+l_{2} c+l_{3} b+0.5 d l_{4}\right)^{-1}\right) \\
- & \left(\left\{n-m+1-l_{1}+l_{3}(a-1)-0.5 l_{4}\right\}\right. \\
& \left.\left.\times\left(C_{1}(x)+l_{2} c+l_{3} b+0.5 d l_{4}\right)^{-1}\right)\right] .
\end{aligned}
$$

The details for the derivation of the Bayes estimates and posterior risks under PLF can be seen from the appendix.

Bayes estimator and corresponding risk under quadratic loss function are, respectively, presented as

$$
\begin{aligned}
& \psi_{\mathrm{QLF}}=\frac{n-m-1-l_{1}+l_{3}(a-1)-0.5 l_{4}}{C_{1}(x)+l_{2} c+l_{3} b+0.5 d l_{4}}, \\
& \rho\left(\psi_{\mathrm{QLF}}\right)=\frac{1}{n-m-l_{1}+l_{3}(a-1)-0.5 l_{4}} .
\end{aligned}
$$

The details for the derivation of the Bayes estimates and posterior risks under QLF can be seen from the appendix.

\section{Bayesian Estimation under Singly Type II Censored Samples}

Under type II censored samples the experiment is terminated after observing some fixed percentage of observations. The likelihood function for the singly type II censored samples can be defined as follows.

Let us observe " $n$ " items for possible failure and only the first " $r$ " failure times have been observed; that is, $x_{1}<$ $x_{2} \cdots<x_{r}$ and remaining " $n-r$ " items are still working. Under the assumptions that the lifetimes of the items are independently and identically distributed random variables 
following Burr type VIII distribution, the likelihood function for " $r$ " observations is

$$
\begin{aligned}
& L(\psi \mid x) \\
& \quad \propto\left\{\prod_{i=1}^{r} f\left(x_{i}, \psi\right)\right\}\left\{1-F\left(x_{r}, \psi\right)\right\}^{n-r}, \\
& L(\psi \mid x) \\
& \propto \sum_{j=0}^{n-r}(-1)^{j}\left(\begin{array}{c}
n-r \\
j
\end{array}\right) \psi^{r} \\
& \quad \times e^{-\psi\left[\sum_{i=1}^{r} \ln \left\{(2 / \pi) \tan ^{-1}\left(e^{t_{i}}\right)\right\}^{-1}+j \ln \left\{(2 / \pi) \tan ^{-1}\left(e^{t_{r}}\right)\right\}^{-1}\right]} .
\end{aligned}
$$

The generalized posterior distribution under singly type II censored samples is

$$
\begin{aligned}
& p(\psi \mid x) \\
& =\left(\sum_{j=0}^{n-r}(-1)^{j}\left(\begin{array}{c}
n-r \\
j
\end{array}\right)\right. \\
& \left.\quad \times \psi^{r-l_{1}+l_{3}(a-1)-0.5 l_{4}} e^{-\psi\left\{C_{2}(x)+l_{2} c+l_{3} b+0.5 d l_{4}\right\}}\right) \\
& \times\left(\Gamma\left(r+1-l_{1}+l_{3}(a-1)-0.5 l_{4}\right)\right. \\
& \quad \times \sum_{j=0}^{n-r}(-1)^{j}\left(\begin{array}{c}
n-r \\
j
\end{array}\right) \\
& \left.\quad \times\left\{C_{2}(x)+l_{2} c+l_{3} b+0.5 d l_{4}\right\}^{-\left(r+1-l_{1}+l_{3}(a-1)-0.5 l_{4}\right)}\right)^{-1} ;
\end{aligned}
$$

where $C_{2}(x)=\sum_{i=1}^{r} \ln \left\{(2 / \pi) \tan ^{-1}\left(e^{x_{i}}\right)\right\}^{-1}+j \ln \{(2 / \pi)$ $\left.\tan ^{-1}\left(e^{x_{r}}\right)\right\}^{-1}$.

Bayes estimator and corresponding risk under LINEX loss function are, respectively, presented as

$$
\begin{array}{r}
\psi_{\mathrm{LLF}}=-\ln \left\{\left(\sum_{j=0}^{n-r}(-1)^{j}\left(\begin{array}{c}
n-r \\
j
\end{array}\right)\right.\right. \\
\times\left\{C_{2}(x)+l_{2} c+l_{3} b+0.5 d l_{4}\right. \\
\left.+1\}^{-\left(r+1-l_{1}+l_{3}(a-1)-0.5 l_{4}\right)}\right)
\end{array}
$$

$$
\begin{aligned}
& \times\left(\sum_{j=0}^{n-r}(-1)^{j}\left(\begin{array}{c}
n-r \\
j
\end{array}\right)\right. \\
& \times\left\{C_{2}(x)+l_{2} c+l_{3} b\right. \\
& \left.\left.\left.+0.5 d l_{4}\right\}^{-\left(r+1-l_{1}+l_{3}(a-1)-0.5 l_{4}\right)}\right)^{-1}\right\}
\end{aligned}
$$$$
\rho\left(\psi_{\text {LLF }}\right)
$$$$
=\left(\left(r+1-l_{1}+l_{3}(a-1)-0.5 l_{4}\right)\right.
$$$$
\times \sum_{j=0}^{n-r}(-1)^{j}\left(\begin{array}{c}
n-r \\
j
\end{array}\right)
$$$$
\times\left\{C_{2}(x)+l_{2} c+l_{3} b\right.
$$$$
\left.\left.+0.5 d l_{4}\right\}^{-\left(r+2-l_{1}+l_{3}(a-1)-0.5 l_{4}\right)}\right)
$$$$
\times\left(\sum_{j=0}^{n-r}(-1)^{j}\left(\begin{array}{c}
n-r \\
j
\end{array}\right)\right.
$$$$
\times\left\{C_{2}(x)+l_{2} c+l_{3} b\right.
$$$$
\left.\left.+0.5 d l_{4}\right\}^{-\left(r+1-l_{1}+l_{3}(a-1)-0.5 l_{4}\right)}\right)^{-1}
$$$$
+\ln \left\{\left(\sum_{j=0}^{n-r}(-1)^{j}\left(\begin{array}{c}
n-r \\
j
\end{array}\right)\right.\right.
$$$$
\times\left\{C_{2}(x)+l_{2} c+l_{3} b+0.5 d l_{4}\right.
$$$$
\left.+1\}^{-\left(r+1-l_{1}+l_{3}(a-1)-0.5 l_{4}\right)}\right)
$$

$$
\times\left(\sum_{j=0}^{n-r}(-1)^{j}\left(\begin{array}{c}
n-r \\
j
\end{array}\right)\right.
$$

$$
\begin{aligned}
& \times\left\{C_{2}(x)+l_{2} c+l_{3} b\right. \\
& \left.\left.\left.\quad+0.5 d l_{4}\right\}^{-\left(r+1-l_{1}+l_{3}(a-1)-0.5 l_{4}\right)}\right)^{-1}\right\} .
\end{aligned}
$$

Bayes estimator and corresponding risk under precautionary loss function are, respectively, presented as

$\psi_{\mathrm{PLF}}$

$$
=\left\{\left(\Gamma\left(r+3-l_{1}+l_{3}(a-1)-0.5 l_{4}\right)\right.\right.
$$




$$
\begin{aligned}
& \times \sum_{j=0}^{n-r}(-1)^{j}\left(\begin{array}{c}
n-r \\
j
\end{array}\right) \\
& \times\left(\sum_{j=0}^{n-r}(-1)^{j}\left(\begin{array}{c}
n-r \\
j
\end{array}\right)\right. \\
& \times\left\{C_{2}(x)+l_{2} c+l_{3} b\right. \\
& \left.\left.+0.5 d l_{4}\right\}^{-\left(r+3-l_{1}+l_{3}(a-1)-0.5 l_{4}\right)}\right) \\
& \times\left\{C_{2}(x)+l_{2} c+l_{3} b\right. \\
& \left.\left.\left.+0.5 d l_{4}\right\}^{-\left(r+1-l_{1}+l_{3}(a-1)-0.5 l_{4}\right)}\right)^{-1}\right) . \\
& \times\left(\Gamma\left(r+1-l_{1}+l_{3}(a-1)-0.5 l_{4}\right)\right. \\
& \times \sum_{j=0}^{n-r}(-1)^{j}\left(\begin{array}{c}
n-r \\
j
\end{array}\right) \\
& \times\left\{C_{2}(x)+l_{2} c+l_{3} b\right. \\
& \left.\left.\left.+0.5 d l_{4}\right\}^{-\left(r+1-l_{1}+l_{3}(a-1)-0.5 l_{4}\right)}\right)^{-1}\right\}^{1 / 2}, \\
& \rho\left(\psi_{\mathrm{PLF}}\right) \\
& =2\left\{\left(\Gamma\left(r+3-l_{1}+l_{3}(a-1)-0.5 l_{4}\right)\right.\right. \\
& \times \sum_{j=0}^{n-r}(-1)^{j}\left(\begin{array}{c}
n-r \\
j
\end{array}\right) \\
& \times\left\{C_{2}(x)+l_{2} c+l_{3} b\right. \\
& \left.\left.+0.5 d l_{4}\right\}^{-\left(r+3-l_{1}+l_{3}(a-1)-0.5 l_{4}\right)}\right) \\
& \times\left(\Gamma\left(r+1-l_{1}+l_{3}(a-1)-0.5 l_{4}\right)\right. \\
& \times \sum_{j=0}^{n-r}(-1)^{j}\left(\begin{array}{c}
n-r \\
j
\end{array}\right) \\
& \times\left\{C_{2}(x)+l_{2} c+l_{3} b\right. \\
& \left.\left.\left.+0.5 d l_{4}\right\}^{-\left(r+1-l_{1}+l_{3}(a-1)-0.5 l_{4}\right)}\right)^{-1}\right\}^{1 / 2} \\
& -\left(\left(2\left(r+1-l_{1}+l_{3}(a-1)-0.5 l_{4}\right)\right.\right. \\
& \times \sum_{j=0}^{n-r}(-1)^{j}\left(\begin{array}{c}
n-r \\
j
\end{array}\right) \\
& \times\left\{C_{2}(x)+l_{2} c+l_{3} b\right. \\
& \left.\left.+0.5 d l_{4}\right\}^{-\left(r+2-l_{1}+l_{3}(a-1)-0.5 l_{4}\right)}\right) \\
& \psi_{\mathrm{QLF}}=\left(\left(r-1-l_{1}+l_{3}(a-1)-0.5 l_{4}\right)\right. \\
& \times \sum_{j=0}^{n-r}(-1)^{j}\left(\begin{array}{c}
n-r \\
j
\end{array}\right) \\
& \times\left\{C_{2}(x)+l_{2} c+l_{3} b\right. \\
& \left.\left.+0.5 d l_{4}\right\}^{-\left(r-l_{1}+l_{3}(a-1)-0.5 l_{4}\right)}\right) \\
& \times\left(\sum_{j=0}^{n-r}(-1)^{j}\left(\begin{array}{c}
n-r \\
j
\end{array}\right)\right. \\
& \times\left\{C_{2}(x)+l_{2} c+l_{3} b\right. \\
& \left.\left.+0.5 d l_{4}\right\}^{-\left(r-1-l_{1}+l_{3}(a-1)-0.5 l_{4}\right)}\right)^{-1}, \\
& \rho\left(\psi_{\mathrm{QLF}}\right)=1-\left(\left[\sum_{j=0}^{n-r}(-1)^{j}\left(\begin{array}{c}
n-r \\
j
\end{array}\right)\right.\right. \\
& \times\left\{C_{2}(t)+l_{2} c+l_{3} b\right. \\
& \left.\left.+0.5 d l_{4}\right\}^{-\left(r+1-l_{1}+l_{3}(a-1)-0.5 l_{4}\right)}\right]^{-1} \\
& \times\left[\Gamma\left(r-l_{1}+l_{3}(a-1)-0.5 l_{4}\right)\right. \\
& \times \sum_{j=0}^{n-r}(-1)^{j}\left(\begin{array}{c}
n-r \\
j
\end{array}\right) \\
& \times\left\{C_{2}(x)+l_{2} c+l_{3} b\right. \\
& \left.\left.\left.+0.5 d l_{4}\right\}^{-\left(r-l_{1}+l_{3}(a-1)-0.5 l_{4}\right)}\right]^{2}\right)
\end{aligned}
$$




$$
\begin{aligned}
& \times\left(\Gamma\left(r+1-l_{1}+l_{3}(a-1)-0.5 l_{4}\right)\right. \\
& \times \Gamma\left(r-1-l_{1}+l_{3}(a-1)-0.5 l_{4}\right) \\
& \times \sum_{j=0}^{n-r}(-1)^{j}\left(\begin{array}{c}
n-r \\
j
\end{array}\right) \\
& \times\left\{C_{2}(x)+l_{2} c+l_{3} b\right. \\
& \left.\left.\quad+0.5 d l_{4}\right\}^{-\left(r-1-l_{1}+l_{3}(a-1)-0.5 l_{4}\right)}\right)^{-1} .
\end{aligned}
$$

\section{Bayesian Estimation under Doubly Type II Censored Samples}

Doubly type II censoring is used when the samples are censored at two test termination points; that is, the observations below and above a particular point cannot be either observed or feasible to be observed. The likelihood function under the doubly type II censored samples can be defined as follows.

Consider a random sample of size " $n$ " from Burr type VIII distribution and let $x_{w}, \ldots, x_{s}$ be the ordered observations that can only be observed. The remaining " $w-1$ " smallest observations and " $n-s$ " largest observations have been censored. Then the likelihood function for the type II doubly censored sample $\underline{x}=\left(x_{w}, \ldots, x_{s}\right)$ can be written as

$$
\begin{aligned}
& L(\psi \mid x) \propto\left\{F\left(x_{w}, \psi\right)\right\}^{w-1} \\
& \times\left\{1-F\left(x_{s}, \psi\right)\right\}^{n-s}\left\{\prod_{i=r}^{s} f\left(x_{i}, \psi\right)\right\}, \\
& L(\psi \mid x) \propto \sum_{j=0}^{n-s}(-1)^{j}\left(\begin{array}{c}
n-s \\
j
\end{array}\right) \psi^{k} \\
& \times e^{-\psi\left[\sum_{i=r}^{s} \ln \left\{(2 / \pi) \tan ^{-1}\left(e^{x_{i}}\right)\right\}^{-1}\right]} \\
& \times e^{-\psi\left[(w-1) \ln \left\{(2 / \pi) \tan ^{-1}\left(e^{x_{w}}\right)\right\}^{-1}\right]} \\
& \times e^{-\psi\left[j \ln \left\{(2 / \pi) \tan ^{-1}\left(e^{x_{s}}\right)\right\}^{-1}\right]} .
\end{aligned}
$$

The generalized posterior distribution under doubly type II censored samples is

$$
\begin{aligned}
p(\psi \mid x)= & \left(\sum_{j=0}^{n-s}(-1)^{j}\left(\begin{array}{c}
n-s \\
j
\end{array}\right)\right. \\
& \times \psi^{k-l_{1}+l_{3}(a-1)-0.5 l_{4}} \\
& \times e^{-\psi\left\{C_{3}(t)+l_{2} c+l_{3} b+0.5 d l_{4}\right\}}
\end{aligned}
$$

$$
\begin{aligned}
& \times\left(\Gamma\left(k+1-l_{1}+l_{3}(a-1)-0.5 l_{4}\right)\right. \\
& \times \sum_{j=0}^{n-s}(-1)^{j}\left(\begin{array}{c}
n-s \\
j
\end{array}\right) \\
& \times\left\{C_{3}(x)+l_{2} c+l_{3} b\right. \\
& \left.\left.\quad+0.5 d l_{4}\right\}^{-\left(k+1-l_{1}+l_{3}(a-1)-0.5 l_{4}\right)}\right)^{-1} ; \\
& \psi>0
\end{aligned}
$$

where $C_{3}(x)=\sum_{i=r}^{s} \ln \left\{(2 / \pi) \tan ^{-1}\left(e^{x_{i}}\right)\right\}^{-1}+(w-$ 1) $\ln \left\{(2 / \pi) \tan ^{-1}\left(e^{x_{w}}\right)\right\}^{-1}+j \ln \left\{(2 / \pi) \tan ^{-1}\left(e^{x_{s}}\right)\right\}^{-1}$. Bayes estimator and corresponding risk under LINEX loss function are, respectively, presented as

$$
\begin{aligned}
& \rho\left(\psi_{\mathrm{LLF}}\right)=\left(\left(k+1-l_{1}+l_{3}(a-1)-0.5 l_{4}\right.\right. \\
& \times \sum_{j=0}^{n-s}(-1)^{j}\left(\begin{array}{c}
n-s \\
j
\end{array}\right) \\
& \times\left\{C_{3}(x)+l_{2} c+l_{3} b\right. \\
& \left.\left.+0.5 d l_{4}\right\}^{-\left(k+2-l_{1}+l_{3}(a-1)-0.5 l_{4}\right)}\right) \\
& \times\left(\sum_{j=0}^{n-s}(-1)^{j}\left(\begin{array}{c}
n-s \\
j
\end{array}\right)\right. \\
& \times\left\{C_{3}(x)+l_{2} c+l_{3} b\right. \\
& \left.\left.+0.5 d l_{4}\right\}^{-\left(k+1-l_{1}+l_{3}(a-1)-0.5 l_{4}\right)}\right)^{-1} \\
& +\ln \left\{\left(\sum_{j=0}^{n-s}(-1)^{j}\left(\begin{array}{c}
n-s \\
j
\end{array}\right)\right.\right. \\
& +\left\{C_{3}(x)+l_{2} c+l_{3} b\right. \\
& \left.\left.+0.5 d l_{4}+1\right\}^{-\left(k+1-l_{1}+l_{3}(a-1)-0.5 l_{4}\right)}\right) \\
& \times\left(\sum_{j=0}^{n-s}(-1)^{j}\left(\begin{array}{c}
n-s \\
j
\end{array}\right)\right.
\end{aligned}
$$




$$
\begin{aligned}
& \times\left\{C_{3}(x)+l_{2} c+l_{3} b\right. \\
& \left.\left.\left.\quad+0.5 d l_{4}\right\}^{-\left(k+1-l_{1}+l_{3}(a-1)-0.5 l_{4}\right)}\right)^{-1}\right\} .
\end{aligned}
$$

Bayes estimator and corresponding risk under precautionary loss function are, respectively, presented as

$$
\begin{aligned}
& \psi_{\mathrm{PLF}}=\left\{\left(\Gamma\left(k+3-l_{1}+l_{3}(a-1)-0.5 l_{4}\right)\right.\right. \\
& \times \sum_{j=0}^{n-s}(-1)^{j}\left(\begin{array}{c}
n-s \\
j
\end{array}\right) \\
& \times\left\{C_{3}(x)+l_{2} c+l_{3} b\right. \\
& \left.\left.+0.5 d l_{4}\right\}^{-\left(k+3-l_{1}+l_{3}(a-1)-0.5 l_{4}\right)}\right) \\
& \times\left(\Gamma\left(k+1-l_{1}+l_{3}(a-1)-0.5 l_{4}\right)\right. \\
& \times \sum_{j=0}^{n-s}(-1)^{j}\left(\begin{array}{c}
n-s \\
j
\end{array}\right) \\
& \times\left\{C_{3}(x)+l_{2} c+l_{3} b\right. \\
& \left.\left.\left.+0.5 d l_{4}\right\}^{-\left(k+1-l_{1}+l_{3}(a-1)-0.5 l_{4}\right)}\right)^{-1}\right\}^{1 / 2}, \\
& \rho\left(\psi_{\mathrm{PLF}}\right)=2\left\{\left(\Gamma\left(k+3-l_{1}+l_{3}(a-1)-0.5 l_{4}\right)\right.\right. \\
& \times \sum_{j=0}^{n-s}(-1)^{j}\left(\begin{array}{c}
n-s \\
j
\end{array}\right) \\
& \times\left\{C_{3}(x)+l_{2} c+l_{3} b\right. \\
& \left.\left.+0.5 d l_{4}\right\}^{-\left(k+3-l_{1}+l_{3}(a-1)-0.5 l_{4}\right)}\right) \\
& \times\left(\Gamma\left(k+1-l_{1}+l_{3}(a-1)-0.5 l_{4}\right)\right. \\
& \times \sum_{j=0}^{n-s}(-1)^{j}\left(\begin{array}{c}
n-s \\
j
\end{array}\right) \\
& \times\left\{C_{3}(x)+l_{2} c+l_{3} b\right. \\
& \left.\left.\left.+0.5 d l_{4}\right\}^{-\left(k+1-l_{1}+l_{3}(a-1)-0.5 l_{4}\right)}\right)^{-1}\right\}^{1 / 2}
\end{aligned}
$$

$$
\begin{aligned}
& -\left(2\left(k+1-l_{1}+l_{3}(a-1)-0.5 l_{4}\right)\right. \\
& \times \sum_{j=0}^{n-s}(-1)^{j}\left(\begin{array}{c}
n-s \\
j
\end{array}\right) \\
& \times\left\{C_{3}(x)+l_{2} c+l_{3} b\right. \\
& \left.\left.+0.5 d l_{4}\right\}^{-\left(k+2-l_{1}+l_{3}(a-1)-0.5 l_{4}\right)}\right) \\
& \times\left(\sum_{j=0}^{n-s}(-1)^{j}\left(\begin{array}{c}
n-s \\
j
\end{array}\right)\right. \\
& \times\left\{C_{3}(x)+l_{2} c+l_{3} b\right. \\
& \left.\left.+0.5 d l_{4}\right\}^{-\left(k+1-l_{1}+l_{3}(a-1)-0.5 l_{4}\right)}\right)^{-1} .
\end{aligned}
$$

Bayes estimator and corresponding risk under quadratic loss function are, respectively, presented as

$$
\begin{aligned}
& \psi_{\mathrm{QLF}}=\left(\left(k-1-l_{1}+l_{3}(a-1)-0.5 l_{4}\right)\right. \\
& \times \sum_{j=0}^{n-s}(-1)^{j}\left(\begin{array}{c}
n-s \\
j
\end{array}\right) \\
& \times\left\{C_{3}(x)+l_{2} c+l_{3} b\right. \\
& \left.\left.+0.5 d l_{4}\right\}^{-\left(k-l_{1}+l_{3}(a-1)-0.5 l_{4}\right)}\right) \\
& \times\left(\sum_{j=0}^{n-s}(-1)^{j}\left(\begin{array}{c}
n-s \\
j
\end{array}\right)\right. \\
& \times\left\{C_{3}(x)+l_{2} c+l_{3} b\right. \\
& \left.\left.+0.5 d l_{4}\right\}^{-\left(k-1-l_{1}+l_{3}(a-1)-0.5 l_{4}\right)}\right)^{-1}, \\
& \rho\left(\psi_{\mathrm{QLF}}\right) \\
& =1-\left(\left[\sum_{j=0}^{n-s}(-1)^{j}\left(\begin{array}{c}
n-s \\
j
\end{array}\right)\right.\right. \\
& \times\left\{C_{3}(t)+l_{2} c+l_{3} b\right. \\
& \left.\left.+0.5 d l_{4}\right\}^{-\left(k+1-l_{1}+l_{3}(a-1)-0.5 l_{4}\right)}\right]^{-1}
\end{aligned}
$$




$$
\begin{aligned}
& \times\left[\Gamma\left(k-l_{1}+l_{3}(a-1)-0.5 l_{4}\right)\right. \\
& \times \sum_{j=0}^{n-s}(-1)^{j}\left(\begin{array}{c}
n-s \\
j
\end{array}\right) \\
& \times\left\{C_{3}(x)+l_{2} c+l_{3} b\right. \\
& \left.\left.\left.+0.5 d l_{4}\right\}^{-\left(k-l_{1}+l_{3}(a-1)-0.5 l_{4}\right)}\right]^{2}\right) \\
& \times\left(\Gamma\left(k+1-l_{1}+l_{3}(a-1)-0.5 l_{4}\right)\right. \\
& \times \Gamma\left(k-1-l_{1}+l_{3}(a-1)-0.5 l_{4}\right) \\
& \times \sum_{j=0}^{n-s}(-1)^{j}\left(\begin{array}{c}
n-s \\
j
\end{array}\right) \\
& \times\left\{C_{3}(x)+l_{2} c+l_{3} b\right. \\
& \left.\left.+0.5 d l_{4}\right\}^{-\left(k-1-l_{1}+l_{3}(a-1)-0.5 l_{4}\right)}\right)^{-1} .
\end{aligned}
$$

\section{Credible Intervals}

Samaniego [14] has discussed that unlike the classical inference, the notion of interval estimation under Bayesian inference is simpler and manages to avoid potential conflicts with the observed data. The posterior distribution of the parameter $\psi$ comprises the basis for all Bayesian inferences about $\psi$. The Bayesian counterpart of a confidence interval for $\psi$ is called a credibility interval for $\psi$ and is obtained from the posterior distribution by selecting an interval corresponding to the probability level desired. For example, any interval $\left(\psi_{L}, \psi_{U}\right)$ for which $\int_{\psi_{L}}^{\psi_{U}} p(\psi \mid x) d \psi=1-\alpha$ is the $100(1-\alpha) \%$ credibility interval for $\psi$, where $p(\psi \mid x)$ is the posterior density of $\theta$ and $\alpha$ is the level of significance. The credibility interval used most often is the central one in which the limits $\left(\psi_{L}, \psi_{U}\right)$ are chosen to satisfy $\int_{-\infty}^{\psi_{L}} p(\psi \mid x) d \psi=$ $\alpha / 2=\int_{\psi_{U}}^{\infty} p(\psi \mid x) d \psi$. Credibility intervals represent the statistician's posterior judgment about intervals that contain $\psi$ with a given probability. They are clearly in harmony with the likelihood principle. So according to the above definition, the $100(1-\alpha) \%$ credible intervals for $\psi$ have been constructed under different censoring techniques as follows.

The generalized credible interval under left censored samples is

$$
\begin{gathered}
\Gamma\left(n-m+1-l_{1}+l_{3}(a-1)-0.5 l_{4},\right. \\
\left.\psi_{L}\left\{C_{1}(x)+l_{2} c+l_{3} b+0.5 d l_{4}\right\}\right)
\end{gathered}
$$

$$
\begin{aligned}
& \times\left(\Gamma\left(n-m+1-l_{1}+l_{3}(a-1)-0.5 l_{4}\right)\right)^{-1} \\
&= 1-\frac{\alpha}{2}, \\
& \Gamma\left(n-m+1-l_{1}+l_{3}(a-1)-0.5 l_{4},\right. \\
&\left.\quad \psi_{U}\left\{C_{1}(x)+l_{2} c+l_{3} b+0.5 d l_{4}\right\}\right) \\
& \quad \times\left(\Gamma\left(n-m+1-l_{1}+l_{3}(a-1)-0.5 l_{4}\right)\right)^{-1} \\
&=\frac{\alpha}{2} .
\end{aligned}
$$

The generalized credible interval under singly type II censored samples is

$$
\begin{aligned}
& \left(\sum _ { j = 0 } ^ { n - r } ( - 1 ) ^ { j } ( \begin{array} { c } 
{ n - r } \\
{ j }
\end{array} ) \left(\Gamma \left(r+1-l_{1}+l_{3}(a-1)-0.5 l_{4},\right.\right.\right. \\
& \psi_{L}\left\{C_{2}(x)+l_{2} c\right. \\
& \left.\left.+l_{3} b+0.5 d l_{4}\right\}\right) \\
& \times\left(\left\{C_{2}(x)+l_{2} c+l_{3} b\right.\right. \\
& \left.\left.\left.+0.5 d l_{4}\right\}^{\left(r+1-l_{1}+l_{3}(a-1)-0.5 l_{4}\right)}\right)^{-1}\right) \\
& \times\left(\sum_{j=0}^{n-r}(-1)^{j}\left(\begin{array}{c}
n-r \\
j
\end{array}\right)\right. \\
& \times\left(\Gamma\left(r+1-l_{1}+l_{3}(a-1)-0.5 l_{4}\right)\right. \\
& \times\left(\left\{C_{2}(x)+l_{2} c+l_{3} b\right.\right. \\
& \left.\left.\left.\left.\left.+0.5 d l_{4}\right\}^{\left(r+1-l_{1}+l_{3}(a-1)-0.5 l_{4}\right)}\right)^{-1}\right)\right)^{-1}\right) \\
& =1-\frac{\alpha}{2}, \\
& \left(\sum _ { j = 0 } ^ { n - r } ( - 1 ) ^ { j } ( \begin{array} { c } 
{ n - r } \\
{ j }
\end{array} ) \left(\Gamma \left(r+1-l_{1}+l_{3}(a-1)\right.\right.\right. \\
& -0.5 l_{4}, \psi_{U}\left\{C_{2}(x)+l_{2} c\right. \\
& \left.\left.+l_{3} b+0.5 d l_{4}\right\}\right) \\
& \times\left(\left\{C_{2}(x)+l_{2} c+l_{3} b\right.\right. \\
& \left.\left.\left.\left.+0.5 d l_{4}\right\}^{\left(r+1-l_{1}+l_{3}(a-1)-0.5 l_{4}\right)}\right)^{-1}\right)\right)
\end{aligned}
$$




$$
\begin{aligned}
& \times\left(\sum_{j=0}^{n-r}(-1)^{j}\left(\begin{array}{c}
n-r \\
j
\end{array}\right)\right. \\
& \quad \times\left(\Gamma\left(r+1-l_{1}+l_{3}(a-1)-0.5 l_{4}\right)\right. \\
& \quad \times\left(\left\{C_{2}(x)+l_{2} c+l_{3} b\right.\right. \\
& \left.\left.\left.\left.+0.5 d l_{4}\right\}^{\left(r+1-l_{1}+l_{3}(a-1)-0.5 l_{4}\right)}\right)^{-1}\right)\right)^{-1} \\
& =\frac{\alpha}{2}
\end{aligned}
$$

The generalized credible interval under doubly type II censored samples is

$$
\begin{aligned}
& \left(\sum_{j=0}^{n-s}(-1)^{j}\left(\begin{array}{c}
n-s \\
j
\end{array}\right)\right. \\
& \times\left(\Gamma \left(k+1-l_{1}+l_{3}(a-1)-0.5 l_{4},\right.\right. \\
& \left.\psi_{L}\left\{C_{3}(x)+l_{2} c+l_{3} b+0.5 d l_{4}\right\}\right) \\
& \times\left(\left\{C_{3}(x)+l_{2} c+l_{3} b\right.\right. \\
& \left.\left.\left.\left.+0.5 d l_{4}\right\}^{\left(k+1-l_{1}+l_{3}(a-1)-0.5 l_{4}\right)}\right)^{-1}\right)\right) \\
& \times\left(\sum_{j=0}^{n-s}(-1)^{j}\left(\begin{array}{c}
n-s \\
j
\end{array}\right)\right. \\
& \times\left(\Gamma\left(k+1-l_{1}+l_{3}(a-1)-0.5 l_{4}\right)\right. \\
& \times\left(\left\{C_{3}(x)+l_{2} c+l_{3} b\right.\right. \\
& \left.\left.\left.\left.+0.5 d l_{4}\right\}^{\left(k+1-l_{1}+l_{3}(a-1)-0.5 l_{4}\right)}\right)^{-1}\right)\right)^{-1} \\
& =1-\frac{\alpha}{2},
\end{aligned}
$$$$
\left(\sum_{j=0}^{n-s}(-1)^{j}\left(\begin{array}{c}
n-s \\
j
\end{array}\right)\right.
$$$$
\times\left(\Gamma \left(k+1-l_{1}+l_{3}(a-1)\right.\right.
$$$$
\left.-0.5 l_{4}, \psi_{U}\left\{C_{3}(x)+l_{2} c+l_{3} b+0.5 d l_{4}\right\}\right)
$$

$$
\begin{aligned}
& \times\left(\left\{C_{3}(x)+l_{2} c+l_{3} b\right.\right. \\
& \left.\left.\left.\left.+0.5 d l_{4}\right\}^{\left(k+1-l_{1}+l_{3}(a-1)-0.5 l_{4}\right)}\right)^{-1}\right)\right) \\
& \times\left(\sum_{j=0}^{n-s}(-1)^{j}\left(\begin{array}{c}
n-s \\
j
\end{array}\right)\right. \\
& \times\left(\Gamma \left(k+1-l_{1}\right.\right. \\
& \left.+l_{3}(a-1)-0.5 l_{4}\right) \\
& \times\left(\left\{C_{3}(x)+l_{2} c+l_{3} b\right.\right. \\
& \left.\left.\left.\left.+0.5 d l_{4}\right\}^{\left(k+1-l_{1}+l_{3}(a-1)-0.5 l_{4}\right)}\right)^{-1}\right)\right)^{-1}
\end{aligned}
$$$$
=\frac{\alpha}{2}
$$

\section{Posterior Predictive Distributions and Intervals}

There are circumstances (e.g., in regression analysis or time series) where out-of-sample (e.g., data at future time points or under different conditions and covariates) predictions are the major interest; such predictions may be in circumstances where the explanatory variates take different values to those actually observed. In clinical trials comparing the efficacy of an established therapy with a new therapy, the interest may be in the predictive probability that a new patient will benefit from the new therapy. The predictive distribution contains the information about the independent future random observation given preceding observations (data at hand). In context of Bayesian inference the predictive distribution is referred to as the posterior predictive distribution. For more illustration, see Bolstad [15], Congdon [16], and Bansal [17]. The posterior predictive distribution can be defined as

$$
g(y \mid x)=\int_{-\infty}^{\infty} p(\psi \mid x) f(y ; \psi) d \psi
$$

where $p(\psi \mid x)$ is the posterior distribution, $f(y ; \psi)$ is density for future observation, and $y=x_{n+1}$ is the future observation given the sample information $x=\left(x_{1}, x_{2}, \ldots, x_{n}\right)$, from model (1). The posterior predictive distributions using different types of censored samples can be derived as follows.

The generalized posterior predictive distribution under left censored samples is

$$
\begin{aligned}
& p(y \mid x) \\
& =\left(\left(n-m+1-l_{1}\right.\right. \\
& \left.\quad+l_{3}(a-1)-0.5 l_{4}\right)
\end{aligned}
$$




$$
\begin{aligned}
& \times e^{y}\left[C_{1}(x)+l_{2} c+l_{3} b\right. \\
& \left.\left.+0.5 d l_{4}\right]^{n-m+1-l_{1}+l_{3}(a-1)-0.5 l_{4}}\right) \\
& \times\left(\left(1+e^{2 y}\right) \tan ^{-1}\left(e^{y}\right)\right. \\
& \times\left[C_{1}(x)+l_{2} c+l_{3} b+0.5 d l_{4}\right)^{-1} ; \\
& \left.\quad-\ln \left\{\frac{2}{\pi} \tan ^{-1}\left(e^{y}\right)\right\}\right]^{n-m+1-l_{1}+l_{3}(a-1)-0.5 l_{4}} \\
& \quad y>0 .
\end{aligned}
$$

The generalized posterior predictive distribution under singly type II censored samples is

$$
\begin{aligned}
p(y \mid x)=( & \left(r+1-l_{1}+l_{3}(a-1)-0.5 l_{4}\right) \\
\times & \times e^{y} \sum_{j=0}^{n-r}(-1)^{j}\left(\begin{array}{c}
n-r \\
j
\end{array}\right) \\
\times & {\left[C_{2}(x)+l_{2} c+l_{3} b+0.5 d l_{4}\right.} \\
& \left.\quad-\ln \left\{\frac{2}{\pi} \tan ^{-1}\left(e^{y}\right)\right\}\right]^{-\left(r+1-l_{1}+l_{3}(a-1)-0.5 l_{4}\right)} \\
\times & \left(1+e^{2 y}\right) \tan ^{-1}\left(e^{y}\right) \\
& \times \sum_{j=0}^{n-r}(-1)^{j}\left(\begin{array}{c}
n-r \\
j
\end{array}\right) \quad y>0 . \\
& \times\left\{C_{2}(x)+l_{2} c+l_{3} b\right. \\
& \left.+0.5 d l_{4}\right\}^{-\left(r+1-l_{1}+l_{3}(a-1)-0.5 l_{4}\right)}
\end{aligned}
$$

The generalized posterior predictive distribution under doubly type II censored samples is

$$
\begin{aligned}
& p(y \mid x)=\left(\left(k+1-l_{1}+l_{3}(a-1)-0.5 l_{4}\right.\right. \\
& \times e^{y} \sum_{j=0}^{n-s}(-1)^{j}\left(\begin{array}{c}
n-s \\
j
\end{array}\right)
\end{aligned}
$$

$$
\begin{aligned}
& \times\left[C_{3}(x)+l_{2} c+l_{3} b+0.5 d l_{4}\right. \\
& \left.\left.-\ln \left\{\frac{2}{\pi} \tan ^{-1}\left(e^{y}\right)\right\}\right]^{-\left(k+1-l_{1}+l_{3}(a-1)-0.5 l_{4}\right)}\right) \\
& \times\left(\left(1+e^{2 y}\right) \tan ^{-1}\left(e^{y}\right)\right. \\
& \times \sum_{j=0}^{n-s}(-1)^{j}\left(\begin{array}{c}
n-s \\
j
\end{array}\right) \\
& \times\left\{C_{3}(x)+l_{2} c+l_{3} b \quad y>0 .\right.
\end{aligned}
$$

The posterior predictive interval can be constructed by solving the following two equations:

$$
\int_{0}^{L} g(y \mid x) d y=\frac{\alpha}{2}, \quad \int_{U}^{\infty} g(y \mid x) d y=\frac{\alpha}{2},
$$

where $\alpha$ is the level of significance.

The posterior predictive intervals cannot be obtained in the closed form, so the approximate solution of the limits has been obtained by iterative procedure.

\section{Simulation Study}

The simulation study has been carried out for $n=$ $20,30,50,70,100$, and 150 using $\psi \in\{2,4,6\}$. As single sample may not fully describe the behavior of the estimators, the results have been replicated sufficiently and average of the results has been presented in Tables 1, 2, 3, 4, 5, 6, and 7. The performance of the different estimates has been compared in terms of magnitude of posterior risks. The posterior risks have been presented in parenthesis in the tables.

It is immediate from Tables $1,2,3,4,5,6$, and 7 that estimated value of the parameter converges to the true value by increasing the sample size. The parameter is overestimated for the majority of the cases. A greater tendency of overestimation is observed for larger values of the parameter. This pattern is similar under each prior, loss function, and censoring scheme. The convergence of the estimated values of the parameter under informative priors is better than those under noninformative priors. In case of loss functions the convergence is better under LLF for smaller values of the parameter, while for larger values of the parameter the convergence is faster under QLF. As far as the censoring techniques are concerned, the better convergence is observed under left censored samples for LLF and PLF, while in case of QLF the estimates under singly type II censored samples have comparatively rapid convergence. 
TABLE 1: Bayes estimators and posterior risks under uniform prior.

\begin{tabular}{|c|c|c|c|c|c|c|c|c|c|}
\hline \multirow{2}{*}{$n$} & \multicolumn{3}{|c|}{ Left censored } & \multicolumn{3}{|c|}{ Singly type II censored } & \multicolumn{3}{|c|}{ Doubly type II censored } \\
\hline & LLF & PLF & QLF & LLF & PLF & QLF & LLF & PLF & QLF \\
\hline \multirow{3}{*}{20} & & & & & $\Psi=2$ & & & & \\
\hline & 2.16360 & 2.24633 & 2.11116 & 2.19235 & 2.26019 & 2.15047 & 2.18079 & 2.25540 & 2.13425 \\
\hline & $(0.09240)$ & $(0.08906)$ & $(0.06250)$ & $(0.07612)$ & $(0.07236)$ & $(0.05409)$ & $(0.08355)$ & $(0.07987)$ & $(0.05465)$ \\
\hline \multirow{2}{*}{30} & 2.10850 & 2.18912 & 2.05740 & 2.13652 & 2.20263 & 2.09571 & 2.12525 & 2.19796 & 2.07990 \\
\hline & $(0.06234)$ & $(0.06009)$ & $(0.04167)$ & $(0.05135)$ & $(0.04882)$ & $(0.03606)$ & $(0.05637)$ & $(0.05389)$ & $(0.03121)$ \\
\hline \multirow{2}{*}{50} & 2.08038 & 2.15993 & 2.02996 & 2.10803 & 2.17326 & 2.06776 & 2.09691 & 2.16865 & 2.05216 \\
\hline & $(0.05368)$ & $(0.05174)$ & $(0.02500)$ & $(0.04422)$ & $(0.04204)$ & $(0.02002)$ & $(0.04854)$ & $(0.04640)$ & $(0.02223)$ \\
\hline \multirow{2}{*}{70} & 2.05895 & 2.13768 & 2.00905 & 2.08631 & 2.15087 & 2.04646 & 2.07531 & 2.14631 & 2.03102 \\
\hline & $(0.04109)$ & $(0.03960)$ & $(0.01786)$ & $(0.03385)$ & $(0.03218)$ & $(0.01545)$ & $(0.03715)$ & $(0.03551)$ & $(0.01337)$ \\
\hline \multirow{2}{*}{100} & 2.01683 & 2.09395 & 1.97424 & 2.04167 & 2.10484 & 2.01100 & 2.02041 & 2.05749 & 1.99583 \\
\hline & $(0.02499)$ & $(0.02496)$ & $(0.01250)$ & $(0.02058)$ & $(0.02029)$ & $(0.01001)$ & $(0.02259)$ & $(0.02239)$ & $(0.01111)$ \\
\hline \multirow{3}{*}{150} & 2.00807 & 2.08485 & 1.96665 & 2.02666 & 2.08937 & 2.00326 & 2.00605 & 2.03264 & 1.98815 \\
\hline & $(0.01644)$ & $(0.01654)$ & $(0.00833)$ & $(0.01354)$ & $(0.01344)$ & $(0.00667)$ & $(0.01486)$ & $(0.01483)$ & $(0.00741)$ \\
\hline & & & & & $\Psi=4$ & & & & \\
\hline \multirow{2}{*}{20} & 4.20964 & 4.37061 & 4.10762 & 4.26559 & 4.39758 & 4.18411 & 4.24309 & 4.38826 & 4.15254 \\
\hline & $(0.15309)$ & $(0.14755)$ & $(0.10355)$ & $(0.12611)$ & $(0.11989)$ & $(0.08961)$ & $(0.13843)$ & $(0.13232)$ & $(0.09054)$ \\
\hline \multirow{2}{*}{30} & 4.18678 & 4.34688 & 4.08531 & 4.24243 & 4.37371 & 4.16139 & 4.22005 & 4.36443 & 4.12999 \\
\hline & $(0.10328)$ & $(0.09955)$ & $(0.06903)$ & $(0.08508)$ & $(0.08089)$ & $(0.05974)$ & $(0.09339)$ & $(0.08928)$ & $(0.05170)$ \\
\hline \multirow{2}{*}{50} & 4.15175 & 4.31051 & 4.05113 & 4.20693 & 4.33711 & 4.12657 & 4.18474 & 4.32791 & 4.09543 \\
\hline & $(0.08894)$ & $(0.08572)$ & $(0.04142)$ & $(0.07326)$ & $(0.06965)$ & $(0.03317)$ & $(0.08042)$ & $(0.07687)$ & $(0.03683)$ \\
\hline \multirow{2}{*}{70} & 4.15016 & 4.30886 & 4.04958 & 4.20532 & 4.33545 & 4.12499 & 4.18314 & 4.32625 & 4.09387 \\
\hline & $(0.06807)$ & $(0.06561)$ & $(0.02959)$ & $(0.05608)$ & $(0.05331)$ & $(0.02560)$ & $(0.06155)$ & $(0.05884)$ & $(0.02216)$ \\
\hline \multirow{2}{*}{100} & 4.07290 & 4.22864 & 4.00663 & 4.12306 & 4.25064 & 4.06113 & 4.08013 & 4.15501 & 4.03049 \\
\hline & $(0.04140)$ & $(0.04135)$ & $(0.02071)$ & $(0.03410)$ & $(0.03362)$ & $(0.01658)$ & $(0.03743)$ & $(0.03710)$ & $(0.01841)$ \\
\hline \multirow{3}{*}{150} & 4.05520 & 4.21026 & 4.00399 & 4.09275 & 4.21940 & 4.04550 & 4.05113 & 4.10483 & 4.01498 \\
\hline & $(0.02724)$ & $(0.02740)$ & $(0.01380)$ & $(0.02243)$ & $(0.02227)$ & $(0.01105)$ & $(0.02462)$ & $(0.02457)$ & $(0.01228)$ \\
\hline & & & & & $\Psi=6$ & & & & \\
\hline \multirow{2}{*}{20} & 6.50207 & 6.75070 & 6.34449 & 6.58849 & 6.79236 & 6.46263 & 6.55374 & 6.77796 & 6.41388 \\
\hline & $(0.29086)$ & $(0.28035)$ & $(0.19674)$ & $(0.23960)$ & $(0.22779)$ & $(0.17026)$ & $(0.26301)$ & $(0.25142)$ & $(0.17202)$ \\
\hline \multirow{2}{*}{30} & 6.46677 & 6.71405 & 6.31004 & 6.55272 & 6.75548 & 6.42754 & 6.51815 & 6.74115 & 6.37905 \\
\hline & $(0.19624)$ & $(0.18915)$ & $(0.13116)$ & $(0.16166)$ & $(0.15369)$ & $(0.11351)$ & $(0.17745)$ & $(0.16962)$ & $(0.09823)$ \\
\hline \multirow{2}{*}{50} & 6.41266 & 6.65787 & 6.25724 & 6.49789 & 6.69896 & 6.37376 & 6.46361 & 6.68475 & 6.32567 \\
\hline & $(0.16898)$ & $(0.16287)$ & $(0.07870)$ & $(0.13920)$ & $(0.13234)$ & $(0.06302)$ & $(0.15280)$ & $(0.14606)$ & $(0.06998)$ \\
\hline \multirow{2}{*}{70} & 6.41020 & 6.65532 & 6.25485 & 6.49540 & 6.69639 & 6.37132 & 6.46114 & 6.68219 & 6.32325 \\
\hline & $(0.12934)$ & $(0.12466)$ & $(0.05621)$ & $(0.10654)$ & $(0.10129)$ & $(0.04865)$ & $(0.11695)$ & $(0.11180)$ & $(0.04210)$ \\
\hline \multirow{2}{*}{100} & 6.29087 & 6.53142 & 6.18852 & 6.36834 & 6.56540 & 6.27268 & 6.30203 & 6.41769 & 6.22537 \\
\hline & $(0.07867)$ & $(0.07857)$ & $(0.03935)$ & $(0.06478)$ & $(0.06387)$ & $(0.03151)$ & $(0.07111)$ & $(0.07048)$ & $(0.03497)$ \\
\hline \multirow{2}{*}{150} & 6.26353 & 6.50303 & 6.18443 & 6.32153 & 6.51714 & 6.24854 & 6.25724 & 6.34018 & 6.20141 \\
\hline & $(0.05175)$ & $(0.05207)$ & $(0.02622)$ & $(0.04262)$ & $(0.04231)$ & $(0.02100)$ & $(0.04678)$ & $(0.04668)$ & $(0.02333)$ \\
\hline
\end{tabular}

The study also depicts that, in terms of posterior risks, the performance of the QLF is the best among all loss functions. The performance of each loss function has been negatively affected by the increasing values of the parameter. The magnitude values of risks associated with estimates using PLF and LLF are close to each other especially in large samples. So, we can replace these two loss functions by each other in the future studies. In comparison of priors, it has been observed that the informative priors have smaller risks than noninformative priors. The estimates based on inverse Levy prior are having the least amounts of posterior risks. The larger choice of the true parametric values has negatively 
TABLE 2: Bayes estimators and posterior risks under Jeffreys prior.

\begin{tabular}{|c|c|c|c|c|c|c|c|c|c|}
\hline \multirow{2}{*}{$n$} & \multicolumn{3}{|c|}{ Left censored } & \multicolumn{3}{|c|}{ Singly type II censored } & \multicolumn{3}{|c|}{ Doubly type II censored } \\
\hline & LLF & PLF & QLF & LLF & PLF & QLF & LLF & PLF & QLF \\
\hline \multirow{3}{*}{20} & & & & & $\Psi=2$ & & & & \\
\hline & 2.15700 & 2.23948 & 2.10472 & 2.18567 & 2.25330 & 2.14392 & 2.17414 & 2.24852 & 2.12774 \\
\hline & $(0.09013)$ & $(0.08687)$ & $(0.06096)$ & $(0.07425)$ & $(0.07059)$ & $(0.05276)$ & $(0.08150)$ & $(0.07791)$ & $(0.05330)$ \\
\hline \multirow{2}{*}{30} & 2.10207 & 2.18245 & 2.05113 & 2.13001 & 2.19592 & 2.08932 & 2.11878 & 2.19126 & 2.07356 \\
\hline & $(0.06081)$ & $(0.05861)$ & $(0.04064)$ & $(0.05009)$ & $(0.04762)$ & $(0.03517)$ & $(0.05499)$ & $(0.05256)$ & $(0.03044)$ \\
\hline \multirow{2}{*}{50} & 2.07404 & 2.15335 & 2.02377 & 2.10161 & 2.16664 & 2.06146 & 2.09052 & 2.16204 & 2.04591 \\
\hline & $(0.05236)$ & $(0.05047)$ & $(0.02439)$ & $(0.04313)$ & $(0.04101)$ & $(0.01953)$ & $(0.04735)$ & $(0.04526)$ & $(0.02168)$ \\
\hline \multirow{2}{*}{70} & 2.05268 & 2.13117 & 2.00293 & 2.07996 & 2.14432 & 2.04022 & 2.06899 & 2.13977 & 2.02483 \\
\hline & $(0.04008)$ & $(0.03863)$ & $(0.01742)$ & $(0.03301)$ & $(0.03139)$ & $(0.01507)$ & $(0.03624)$ & $(0.03464)$ & $(0.01304)$ \\
\hline \multirow{2}{*}{100} & 2.01068 & 2.08757 & 1.96822 & 2.03545 & 2.09843 & 2.00487 & 2.01425 & 2.05122 & 1.98975 \\
\hline & $(0.02438)$ & $(0.02435)$ & $(0.01219)$ & $(0.02007)$ & $(0.01979)$ & $(0.00976)$ & $(0.02204)$ & $(0.02184)$ & $(0.01084)$ \\
\hline \multirow{3}{*}{150} & 2.00195 & 2.07850 & 1.96066 & 2.02049 & 2.08301 & 1.99716 & 1.99994 & 2.02645 & 1.98209 \\
\hline & $(0.01604)$ & $(0.01613)$ & $(0.00813)$ & $(0.01321)$ & $(0.01311)$ & $(0.00651)$ & $(0.01449)$ & $(0.01447)$ & $(0.00723)$ \\
\hline & & & & & $\Psi=4$ & & & & \\
\hline \multirow{2}{*}{20} & 4.19681 & 4.35729 & 4.09510 & 4.25259 & 4.38418 & 4.17136 & 4.23016 & 4.37488 & 4.13988 \\
\hline & $(0.14933)$ & $(0.14393)$ & $(0.10101)$ & $(0.12301)$ & $(0.11695)$ & $(0.08741)$ & $(0.13503)$ & $(0.12907)$ & $(0.08831)$ \\
\hline \multirow{2}{*}{30} & 4.17403 & 4.33363 & 4.07286 & 4.22950 & 4.36038 & 4.14870 & 4.20719 & 4.35113 & 4.11741 \\
\hline & $(0.10075)$ & $(0.09711)$ & $(0.06734)$ & $(0.08299)$ & $(0.07890)$ & $(0.05827)$ & $(0.09110)$ & $(0.08708)$ & $(0.05043)$ \\
\hline \multirow{2}{*}{50} & 4.13910 & 4.29737 & 4.03879 & 4.19411 & 4.32389 & 4.11399 & 4.17199 & 4.31472 & 4.08295 \\
\hline & $(0.08675)$ & $(0.08362)$ & $(0.04040)$ & $(0.07146)$ & $(0.06794)$ & $(0.03235)$ & $(0.07844)$ & $(0.07499)$ & $(0.03593)$ \\
\hline \multirow{2}{*}{70} & 4.13752 & 4.29573 & 4.03724 & 4.19251 & 4.32224 & 4.11242 & 4.17039 & 4.31307 & 4.08139 \\
\hline & $(0.06640)$ & $(0.06400)$ & $(0.02886)$ & $(0.05470)$ & $(0.05200)$ & $(0.02497)$ & $(0.06004)$ & $(0.05739)$ & $(0.02161)$ \\
\hline \multirow{2}{*}{100} & 4.06049 & 4.21575 & 3.99442 & 4.11049 & 4.23769 & 4.04875 & 4.06770 & 4.14235 & 4.01821 \\
\hline & $(0.04039)$ & $(0.04034)$ & $(0.02020)$ & $(0.03326)$ & $(0.03279)$ & $(0.01618)$ & $(0.03651)$ & $(0.03618)$ & $(0.01795)$ \\
\hline \multirow{3}{*}{150} & 4.04284 & 4.19743 & 3.99179 & 4.08028 & 4.20654 & 4.03317 & 4.03879 & 4.09232 & 4.00275 \\
\hline & $(0.02657)$ & $(0.02673)$ & $(0.01346)$ & $(0.02188)$ & $(0.02172)$ & $(0.01078)$ & $(0.02402)$ & $(0.02397)$ & $(0.01198)$ \\
\hline & & & & & $\Psi=6$ & & & & \\
\hline \multirow{2}{*}{20} & 6.48226 & 6.73013 & 6.32516 & 6.56842 & 6.77167 & 6.44294 & 6.53377 & 6.75730 & 6.39433 \\
\hline & $(0.28372$ & $(0.27346)$ & $(0.19191)$ & $(0.23372)$ & $(0.22220)$ & $(0.16608)$ & $(0.25655)$ & $(0.24524)$ & $(0.16779)$ \\
\hline \multirow{2}{*}{30} & 6.44706 & 6.69359 & 6.29081 & 6.53275 & 6.73489 & 6.40795 & 6.49829 & 6.72061 & 6.35961 \\
\hline & $(0.19142$ & $(0.18450)$ & $(0.12794)$ & $(0.15768)$ & $(0.14991)$ & $(0.11072)$ & $(0.17309)$ & $(0.16546)$ & $(0.09582)$ \\
\hline \multirow{2}{*}{50} & 6.39312 & 6.63758 & 6.23817 & 6.47809 & 6.67854 & 6.35434 & 6.44391 & 6.66438 & 6.30640 \\
\hline & $(0.16483$ & $(0.15887)$ & $(0.07676)$ & $(0.13578)$ & $(0.12909)$ & $(0.06147)$ & $(0.14905)$ & $(0.14247)$ & $(0.06826)$ \\
\hline \multirow{2}{*}{70} & 6.32657 & 6.56848 & 6.17324 & 6.41065 & 6.60902 & 6.28819 & 6.37684 & 6.59500 & 6.24075 \\
\hline & $(0.12616$ & $(0.12160)$ & $(0.05483)$ & $(0.10393)$ & $(0.09880)$ & $(0.04745)$ & $(0.11408)$ & $(0.10905)$ & $(0.04106)$ \\
\hline \multirow{2}{*}{100} & 6.14588 & 6.38089 & 6.04589 & 6.22157 & 6.41409 & 6.12811 & 6.15679 & 6.26978 & 6.08189 \\
\hline & $(0.07673$ & $(0.07664)$ & $(0.03838)$ & $(0.06319)$ & $(0.06230)$ & $(0.03074)$ & $(0.06936)$ & $(0.06875)$ & $(0.03411)$ \\
\hline \multirow{2}{*}{150} & 6.07266 & 6.30486 & 6.03308 & 6.12889 & 6.31854 & 6.05813 & 6.06656 & 6.14697 & 6.01243 \\
\hline & $(0.05048$ & $(0.05079)$ & $(0.02558)$ & $(0.04158)$ & $(0.04127)$ & $(0.02048)$ & $(0.04563)$ & $(0.04554)$ & $(0.02275)$ \\
\hline
\end{tabular}

affected the performance of each prior. The increased censoring rate results in slower convergence of the estimates and the inflated posterior risks. It is a natural consequence of the censoring that can be prevented by increased sample size. It is interesting to note that, for each prior and loss function, the posterior risks under singly type II censoring are smaller than those under other censoring techniques.

The Bayesian interval estimation exhibited a similar pattern as observed in case of point estimation. All the credible intervals are skewed toward the right. The credible 
TABLE 3: Bayes estimators and posterior risks under exponential prior.

\begin{tabular}{|c|c|c|c|c|c|c|c|c|c|}
\hline \multirow{2}{*}{$n$} & \multicolumn{3}{|c|}{ Left censored } & \multicolumn{3}{|c|}{ Singly type II censored } & \multicolumn{3}{|c|}{ Doubly type II censored } \\
\hline & LLF & PLF & QLF & LLF & PLF & QLF & LLF & PLF & QLF \\
\hline & & & & & $\Psi=2$ & & & & \\
\hline \multirow{2}{*}{20} & 2.13477 & 2.21640 & 2.08304 & 2.16315 & 2.23008 & 2.12182 & 2.15174 & 2.22535 & 2.10582 \\
\hline & $(0.08792)$ & $(0.08474)$ & $(0.05947)$ & $(0.07242)$ & $(0.06885)$ & $(0.05146)$ & $(0.07950)$ & $(0.07599)$ & $(0.05199)$ \\
\hline \multirow{2}{*}{30} & 2.08041 & 2.15996 & 2.02999 & 2.10806 & 2.17329 & 2.06779 & 2.09694 & 2.16868 & 2.05219 \\
\hline & $(0.05931)$ & $(0.05717)$ & $(0.03964)$ & $(0.04886)$ & $(0.04645)$ & $(0.03431)$ & $(0.05363)$ & $(0.05127)$ & $(0.02969)$ \\
\hline \multirow{2}{*}{50} & 2.05267 & 2.13116 & 2.00292 & 2.07995 & 2.14431 & 2.04022 & 2.06898 & 2.13976 & 2.02482 \\
\hline & $(0.05108)$ & $(0.04923)$ & $(0.02379)$ & $(0.04207)$ & $(0.04000)$ & $(0.01905)$ & $(0.04618)$ & $(0.04415)$ & $(0.02115)$ \\
\hline \multirow{2}{*}{70} & 2.03152 & 2.10920 & 1.98229 & 2.05852 & 2.12222 & 2.01920 & 2.04766 & 2.11772 & 2.00397 \\
\hline & $(0.03909)$ & $(0.03768)$ & $(0.01699)$ & $(0.03220)$ & $(0.03062)$ & $(0.01470)$ & $(0.03535)$ & $(0.03379)$ & $(0.01272)$ \\
\hline \multirow{2}{*}{100} & 1.98996 & 2.06606 & 1.94794 & 2.01447 & 2.07681 & 1.98421 & 1.99350 & 2.03008 & 1.96924 \\
\hline & $(0.02378)$ & $(0.02375)$ & $(0.01189)$ & $(0.01958)$ & $(0.01931)$ & $(0.00952)$ & $(0.02149)$ & $(0.02130)$ & $(0.01057)$ \\
\hline \multirow{2}{*}{150} & 1.98132 & 2.05708 & 1.94045 & 1.99966 & 2.06154 & 1.97658 & 1.97933 & 2.00556 & 1.96167 \\
\hline & $(0.01564)$ & $(0.01574)$ & $(0.00793)$ & $(0.01288)$ & $(0.01279)$ & $(0.00635)$ & $(0.01414)$ & $(0.01411)$ & $(0.00705)$ \\
\hline \multirow{3}{*}{20} & & & & & $\Psi=4$ & & & & \\
\hline & 4.15357 & 4.31239 & 4.05290 & 4.20877 & 4.33901 & 4.12837 & 4.18657 & 4.32980 & 4.09722 \\
\hline & $(0.14566)$ & $(0.14039)$ & $(0.09852)$ & $(0.11999)$ & $(0.11407)$ & $(0.08526)$ & $(0.13171)$ & $(0.12590)$ & $(0.08614)$ \\
\hline \multirow{2}{*}{30} & 4.13101 & 4.28898 & 4.03089 & 4.18592 & 4.31544 & 4.10595 & 4.16384 & 4.30629 & 4.07498 \\
\hline & $(0.09827)$ & $(0.09472)$ & $(0.06568)$ & $(0.08095)$ & $(0.07696)$ & $(0.05684)$ & $(0.08886)$ & $(0.08494)$ & $(0.04919)$ \\
\hline \multirow{2}{*}{50} & 4.09645 & 4.25309 & 3.99717 & 4.15089 & 4.27934 & 4.07160 & 4.12900 & 4.27026 & 4.04088 \\
\hline & $(0.08462)$ & $(0.08156)$ & $(0.03941)$ & $(0.06971)$ & $(0.06627)$ & $(0.03156)$ & $(0.07652)$ & $(0.07314)$ & $(0.03504)$ \\
\hline \multirow{2}{*}{70} & 4.09488 & 4.25146 & 3.99564 & 4.14930 & 4.27770 & 4.07004 & 4.12742 & 4.26862 & 4.03933 \\
\hline & $(0.06477)$ & $(0.06243)$ & $(0.02815)$ & $(0.05335)$ & $(0.05072)$ & $(0.02436)$ & $(0.05857)$ & $(0.05598)$ & $(0.02108)$ \\
\hline \multirow{2}{*}{100} & 4.01865 & 4.17231 & 3.95326 & 4.06814 & 4.19402 & 4.00703 & 4.02578 & 4.09966 & $3.97680)$ \\
\hline & $(0.03939)$ & $(0.03935)$ & $(0.01970)$ & $(0.03244)$ & $(0.03198)$ & $(0.01578)$ & $(0.03561)$ & $(0.03530)$ & $(0.01751)$ \\
\hline \multirow{3}{*}{150} & 4.00118 & 4.15418 & 3.95066 & 4.03824 & 4.16319 & 3.99161 & 3.99717 & 4.05015 & 3.96150 \\
\hline & $(0.02592)$ & $(0.02607)$ & $(0.01313)$ & $(0.02134)$ & $(0.02119)$ & $(0.01051)$ & $(0.02343)$ & $(0.02338)$ & $(0.01168)$ \\
\hline & & & & & $\Psi=6$ & & & & \\
\hline \multirow{2}{*}{20} & 6.41546 & 6.66078 & 6.25998 & 6.50073 & 6.70189 & 6.37654 & 6.46644 & 6.68767 & 6.32844 \\
\hline & $(0.27675)$ & $(0.26675)$ & $(0.18720)$ & $(0.22798)$ & $(0.21674)$ & $(0.16200)$ & $(0.25025)$ & $(0.23922)$ & $(0.16367)$ \\
\hline \multirow{2}{*}{30} & 6.38063 & 6.62461 & 6.22599 & 6.46543 & 6.66549 & 6.34192 & 6.43132 & 6.65135 & 6.29407 \\
\hline & $(0.18672)$ & $(0.17997)$ & $(0.12480)$ & $(0.15381)$ & $(0.14623)$ & $(0.10800)$ & $(0.16884)$ & $(0.16139)$ & $(0.09346)$ \\
\hline \multirow{2}{*}{50} & 6.32724 & 6.56918 & 6.17389 & 6.41133 & 6.60972 & 6.28886 & 6.37751 & 6.59570 & 6.24141 \\
\hline & $(0.16078)$ & $(0.15497)$ & $(0.07488)$ & $(0.13244)$ & $(0.12592)$ & $(0.05996)$ & $(0.14538)$ & $(0.13897)$ & $(0.06658)$ \\
\hline \multirow{2}{*}{70} & 6.26137 & 6.50080 & 6.10962 & 6.34459 & 6.54092 & 6.22339 & 6.31113 & 6.52704 & 6.17644 \\
\hline & $(0.12306)$ & $(0.11861)$ & $(0.05348)$ & $(0.10137)$ & $(0.09638)$ & $(0.04629)$ & $(0.11128)$ & $(0.10637)$ & $(0.04006)$ \\
\hline \multirow{2}{*}{100} & 6.08255 & 6.31513 & 5.98359 & 6.15746 & 6.34799 & 6.06497 & 6.09335 & 6.20517 & 6.01921 \\
\hline & $(0.07485)$ & $(0.07476)$ & $(0.03744)$ & $(0.06164)$ & $(0.06077)$ & $(0.02998)$ & $(0.06766)$ & $(0.06706)$ & $(0.03328)$ \\
\hline \multirow{2}{*}{150} & 6.01008 & 6.23989 & 5.97091 & 6.06574 & 6.25343 & 5.99570 & 6.00405 & 6.08363 & 5.95047 \\
\hline & $(0.04924)$ & $(0.04954)$ & $(0.02495)$ & $(0.04055)$ & $(0.04025)$ & $(0.01998)$ & $(0.04451)$ & $(0.04442)$ & $(0.02219)$ \\
\hline
\end{tabular}

intervals under informative priors are more specific than those under noninformative priors on the basis of each censoring technique. The minimum widths of credible intervals have been observed under inverse Levy prior. In addition, the credible intervals under singly type II censored samples have smaller widths as compared to other censoring techniques. Similar trends have been observed as far as the posterior predictive intervals are concerned. 
TABLE 4: Bayes estimators and posterior risks under gamma prior.

\begin{tabular}{|c|c|c|c|c|c|c|c|c|c|}
\hline \multirow{2}{*}{$n$} & \multicolumn{3}{|c|}{ Left censored } & \multicolumn{3}{|c|}{ Singly type II censored } & \multicolumn{3}{|c|}{ Doubly type II censored } \\
\hline & LLF & PLF & QLF & LLF & PLF & QLF & LLF & PLF & QLF \\
\hline & \multicolumn{9}{|c|}{$\Psi=2$} \\
\hline \multirow{2}{*}{20} & 2.17527 & 2.25845 & 2.12255 & 2.20418 & 2.27239 & 2.16207 & 2.19255 & 2.26757 & 2.14576 \\
\hline & $(0.07926)$ & $(0.07640)$ & $(0.05361)$ & $(0.06529)$ & $(0.06207)$ & $(0.04640)$ & $(0.07167)$ & $(0.06851)$ & $(0.04688)$ \\
\hline \multirow{2}{*}{30} & 2.11988 & 2.20094 & 2.06850 & 2.14805 & 2.21452 & 2.10702 & 2.13672 & 2.20982 & 2.09112 \\
\hline & $(0.05347)$ & $(0.05154)$ & $(0.03574)$ & $(0.04405)$ & $(0.04188)$ & $(0.03093)$ & $(0.04835)$ & $(0.04622)$ & $(0.02677)$ \\
\hline \multirow{2}{*}{50} & 2.09161 & 2.17159 & 2.04091 & 2.11941 & 2.18499 & 2.07892 & 2.10823 & 2.18035 & 2.06323 \\
\hline & $(0.04605)$ & $(0.04438)$ & $(0.02144)$ & $(0.03793)$ & $(0.03606)$ & $(0.01717)$ & $(0.04164)$ & $(0.03980)$ & $(0.01907)$ \\
\hline \multirow{2}{*}{70} & 2.07006 & 2.14922 & 2.01989 & 2.09757 & 2.16248 & 2.05750 & 2.08651 & 2.15789 & 2.04198 \\
\hline & $(0.03524)$ & $(0.03397)$ & $(0.01532)$ & $(0.02903)$ & $(0.02760)$ & $(0.01326)$ & $(0.03187)$ & $(0.03046)$ & $(0.01147)$ \\
\hline \multirow{2}{*}{100} & 2.02771 & 2.10525 & 1.98489 & 2.05268 & 2.11620 & 2.02185 & 2.03131 & 2.06859 & 2.00660 \\
\hline & $(0.02144)$ & $(0.02141)$ & $(0.01072)$ & $(0.01765)$ & $(0.01740)$ & $(0.00859)$ & $(0.01938)$ & $(0.01921)$ & $(0.00953)$ \\
\hline \multirow{3}{*}{150} & 2.01890 & 2.09610 & 1.97726 & 2.03760 & 2.10065 & 2.01407 & 2.01687 & 2.04361 & 1.99888 \\
\hline & $(0.01410)$ & $(0.01419)$ & $(0.00715)$ & $(0.01161)$ & $(0.01153)$ & $(0.00572)$ & $(0.01275)$ & $(0.01272)$ & $(0.00636)$ \\
\hline & & & & & $\Psi=4$ & & & & \\
\hline \multirow{2}{*}{20} & 4.23236 & 4.39420 & 4.12978 & 4.28861 & 4.42131 & 4.20668 & 4.26599 & 4.41194 & 4.17495 \\
\hline & $(0.13132)$ & $(0.12657)$ & $(0.08882)$ & $(0.10817)$ & $(0.10284)$ & $(0.07687)$ & $(0.11874)$ & $(0.11351)$ & $(0.07766)$ \\
\hline \multirow{2}{*}{30} & 4.20938 & 4.37034 & 4.10736 & 4.26532 & 4.39731 & 4.18384 & 4.24282 & 4.38798 & 4.15228 \\
\hline & $(0.08860)$ & $(0.08539)$ & $(0.05922)$ & $(0.07298)$ & $(0.06938)$ & $(0.05125)$ & $(0.08011)$ & $(0.07658)$ & $(0.04435)$ \\
\hline \multirow{2}{*}{50} & 4.17416 & 4.33377 & 4.07299 & 4.22963 & 4.36051 & 4.14883 & 4.20732 & 4.35126 & 4.11753 \\
\hline & $(0.07629)$ & $(0.07353)$ & $(0.03553)$ & $(0.06284)$ & $(0.05975)$ & $(0.02845)$ & $(0.06898)$ & $(0.06594)$ & $(0.03159)$ \\
\hline \multirow{2}{*}{70} & 4.17256 & 4.33211 & 4.07143 & 4.22801 & 4.35884 & 4.14725 & 4.20571 & 4.34960 & 4.11596 \\
\hline & $(0.05839)$ & $(0.05628)$ & $(0.02538)$ & $(0.04810)$ & $(0.04573)$ & $(0.02196)$ & $(0.05280)$ & $(0.05047)$ & $(0.01901)$ \\
\hline \multirow{2}{*}{100} & 4.09488 & 4.25146 & 4.02825 & 4.14531 & 4.27358 & 4.08304 & 4.10215 & 4.17743 & 4.05224 \\
\hline & $(0.03552)$ & $(0.03547)$ & $(0.01776)$ & $(0.02925)$ & $(0.02884)$ & $(0.01423)$ & $(0.03210)$ & $(0.03182)$ & $(0.01579)$ \\
\hline \multirow{3}{*}{150} & 4.07708 & 4.23298 & 4.02560 & 4.11484 & 4.24217 & 4.06733 & 4.07299 & 4.12698 & 4.03665 \\
\hline & $(0.02336)$ & $(0.02351)$ & $(0.01184)$ & $(0.01924)$ & $(0.01910)$ & $(0.00948)$ & $(0.02112)$ & $(0.02108)$ & $(0.01053)$ \\
\hline & & & & & $\Psi=6$ & & & & \\
\hline \multirow{2}{*}{20} & 6.53716 & 6.78713 & 6.37873 & 6.62405 & 6.82902 & 6.49750 & 6.58910 & 6.81453 & 6.44849 \\
\hline & $(0.24950)$ & $(0.24048)$ & $(0.16877)$ & $(0.20553)$ & $(0.19540)$ & $(0.14605)$ & $(0.22561)$ & $(0.21566)$ & $(0.14756)$ \\
\hline \multirow{2}{*}{30} & 6.50166 & 6.75028 & 6.34409 & 6.58808 & 6.79193 & 6.46222 & 6.55332 & 6.77753 & 6.41347 \\
\hline & $(0.16833)$ & $(0.16225)$ & $(0.11251)$ & $(0.13867)$ & $(0.13183)$ & $(0.09737)$ & $(0.15221)$ & $(0.14550)$ & $(0.08426)$ \\
\hline \multirow{2}{*}{50} & 6.44726 & 6.69379 & 6.29101 & 6.53295 & 6.73511 & 6.40815 & 6.49849 & 6.72082 & 6.35981 \\
\hline & $(0.14495)$ & $(0.13971)$ & $(0.06751)$ & $(0.11940)$ & $(0.11352)$ & $(0.05406)$ & $(0.13107)$ & $(0.12529)$ & $(0.06003)$ \\
\hline \multirow{2}{*}{70} & 6.38015 & 6.62412 & 6.22552 & 6.46495 & 6.66500 & 6.34145 & 6.43084 & 6.65086 & 6.29360 \\
\hline & $(0.11094)$ & $(0.10693)$ & $(0.04822)$ & $(0.09139)$ & $(0.08689)$ & $(0.04173)$ & $(0.10032)$ & $(0.09590)$ & $(0.03611)$ \\
\hline \multirow{2}{*}{100} & 6.19793 & 6.43493 & 6.09709 & 6.27426 & 6.46841 & 6.18002 & 6.20893 & 6.32288 & 6.13340 \\
\hline & $(0.06748)$ & $(0.06740)$ & $(0.03375)$ & $(0.05557)$ & $(0.05479)$ & $(0.02703)$ & $(0.06100)$ & $(0.06046)$ & $(0.03000)$ \\
\hline \multirow{2}{*}{150} & 6.12409 & 6.35826 & 6.08418 & 6.18080 & 6.37206 & 6.10943 & 6.11794 & 6.19903 & 6.06335 \\
\hline & $(0.04439)$ & $(0.04466)$ & $(0.02249)$ & $(0.03656)$ & $(0.03629)$ & $(0.01801)$ & $(0.04013)$ & $(0.04004)$ & (0.02001) \\
\hline
\end{tabular}

\section{Real Life Example}

This section covers the analysis of real life data set regarding the breaking strengths of 64 single carbon fibers of length 10 , presented by Lawless [18]. The idea has been to see whether the results and properties of the Bayes estimators, explored by simulation study, are applicable to a real life situation. The amounts of posterior risks associated with each estimate have been presented in parenthesis in Tables 8, 9, and 10 .
The results regarding the analysis of the real life data displayed the characteristics of the estimates parallel to those observed under simulation study. The inverse Levy prior has been found to be the most suitable prior and QLF the best working loss function. The results under real life data also indicated that the estimates under singly type II censored samples are more efficient under each prior and loss function. So, the information lost from the right end may not be as serious as from the left tail. 
TABLE 5: Bayes estimators and posterior risks under inverse Levy prior.

\begin{tabular}{|c|c|c|c|c|c|c|c|c|c|}
\hline \multirow{2}{*}{$n$} & \multicolumn{3}{|c|}{ Left censored } & \multicolumn{3}{|c|}{ Singly type II censored } & \multicolumn{3}{|c|}{ Doubly type II censored } \\
\hline & LLF & PLF & QLF & LLF & PLF & QLF & LLF & PLF & QLF \\
\hline & \multicolumn{9}{|c|}{$\Psi=2$} \\
\hline \multirow{2}{*}{20} & 2.15285 & 2.23518 & 2.10068 & 2.18147 & 2.24897 & 2.13980 & 2.16996 & 2.24420 & 2.12365 \\
\hline & $(0.07731)$ & $(0.07452)$ & $(0.05230)$ & $(0.06369)$ & $(0.06055)$ & $(0.04526)$ & $(0.06991)$ & $(0.06683)$ & $(0.04572)$ \\
\hline \multirow{2}{*}{30} & 2.09803 & 2.17826 & 2.04718 & 2.12592 & 2.19170 & 2.08531 & 2.11470 & 2.18705 & 2.06957 \\
\hline & $(0.05216)$ & $(0.05028)$ & $(0.03486)$ & $(0.04297)$ & $(0.04085)$ & $(0.03017)$ & $(0.04717)$ & $(0.04509)$ & $(0.026110)$ \\
\hline \multirow{2}{*}{50} & 2.07005 & 2.14921 & 2.01988 & 2.09757 & 2.16247 & 2.05750 & 2.08650 & 2.15788 & 2.04197 \\
\hline & $(0.04492)$ & $(0.04329)$ & $(0.02092)$ & $(0.03700)$ & $(0.03518)$ & $(0.01675)$ & $(0.04061)$ & $(0.03882)$ & $(0.01860)$ \\
\hline \multirow{2}{*}{70} & 2.04873 & 2.12707 & 1.99908 & 2.07596 & 2.14020 & 2.03630 & 2.06501 & 2.13566 & 2.02094 \\
\hline & $(0.03438)$ & $(0.03314)$ & $(0.01494)$ & $(0.02832)$ & $(0.02692)$ & $(0.01293)$ & $(0.03109)$ & $(0.02972)$ & $(0.01119)$ \\
\hline \multirow{2}{*}{100} & 2.00682 & 2.08355 & 1.96444 & 2.03153 & 2.09440 & 2.00102 & 2.01038 & 2.04728 & 1.98592 \\
\hline & $(0.02091)$ & $(0.02088)$ & $(0.01046)$ & $(0.01722)$ & $(0.01698)$ & $(0.00838)$ & $(0.01890)$ & $(0.01873)$ & $(0.00930)$ \\
\hline \multirow{3}{*}{150} & 2.00673 & 2.08346 & 1.96534 & 2.02531 & 2.08798 & 2.00192 & 2.00471 & 2.03128 & 1.98682 \\
\hline & $(0.01376)$ & $(0.01384)$ & $(0.00697)$ & $(0.01133)$ & $(0.01125)$ & $(0.00558)$ & $(0.01243)$ & $(0.01241)$ & $(0.00620)$ \\
\hline & & & & & $\Psi=4$ & & & & \\
\hline \multirow{2}{*}{20} & 4.18874 & 4.34891 & 4.08723 & 4.24442 & 4.37575 & 4.16333 & 4.22203 & 4.36647 & 4.13193 \\
\hline & $(0.12809)$ & $(0.12346)$ & $(0.08664)$ & $(0.10552)$ & $(0.10032)$ & $(0.07498)$ & $(0.11583)$ & $(0.11072)$ & $(0.07575)$ \\
\hline \multirow{2}{*}{30} & 4.16600 & 4.32530 & 4.06503 & 4.22137 & 4.35199 & 4.14073 & 4.19910 & 4.34276 & 4.10949 \\
\hline & $(0.08642)$ & $(0.08330)$ & $(0.05776)$ & $(0.07119)$ & $(0.06768)$ & $(0.04999)$ & $(0.07814)$ & $(0.07470)$ & $(0.04326)$ \\
\hline \multirow{2}{*}{50} & 4.13114 & 4.28911 & 4.03102 & 4.18605 & 4.31558 & 4.10608 & 4.16397 & 4.30643 & 4.07510 \\
\hline & $(0.07441)$ & $(0.07173)$ & $(0.03466)$ & $(0.06130)$ & $(0.05828)$ & $(0.02775)$ & $(0.06729)$ & $(0.06432)$ & $(0.03082)$ \\
\hline \multirow{2}{*}{70} & 4.12956 & 4.28747 & 4.02948 & 4.18445 & 4.31393 & 4.10451 & 4.16237 & 4.30478 & 4.07354 \\
\hline & $(0.05696)$ & $(0.05490)$ & $(0.02475)$ & $(0.04692)$ & $(0.04461)$ & $(0.02142)$ & $(0.05150)$ & $(0.04923)$ & $(0.01854)$ \\
\hline \multirow{2}{*}{100} & 4.05268 & 4.20765 & 4.02703 & 4.10259 & 4.22954 & 4.07060 & 4.05988 & 4.13439 & 4.03989 \\
\hline & $(0.03464)$ & $(0.03460)$ & $(0.01733)$ & $(0.02853)$ & $(0.02813)$ & $(0.01388)$ & $(0.03132)$ & $(0.03104)$ & $(0.01540)$ \\
\hline \multirow{3}{*}{150} & 4.05250 & 4.20746 & 4.00132 & 4.09003 & 4.21659 & 4.04280 & 4.04843 & 4.10209 & 4.01231 \\
\hline & $(0.02279)$ & $(0.02293)$ & $(0.01155)$ & $(0.01877)$ & $(0.01863)$ & $(0.00925)$ & $(0.02060)$ & $(0.02056)$ & $(0.01027)$ \\
\hline & & & & & $\Psi=6$ & & & & \\
\hline \multirow{2}{*}{20} & 6.46980 & 6.71719 & 6.31300 & 6.55579 & 6.75865 & 6.43055 & 6.52120 & 6.74431 & 6.38204 \\
\hline & $(0.24337)$ & $(0.23458)$ & $(0.16462)$ & $(0.20048)$ & $(0.19060)$ & $(0.14246)$ & $(0.22007)$ & $(0.21037)$ & $(0.14393)$ \\
\hline \multirow{2}{*}{30} & 6.43467 & 6.68072 & 6.27872 & 6.52019 & 6.72195 & 6.39563 & 6.48579 & 6.70769 & 6.34738 \\
\hline & $(0.16420)$ & $(0.15826)$ & $(0.10975)$ & $(0.13526)$ & $(0.12859)$ & $(0.09498)$ & $(0.14847)$ & $(0.14193)$ & $(0.08219)$ \\
\hline \multirow{2}{*}{50} & 6.38083 & 6.62482 & 6.22618 & 6.46563 & 6.66570 & 6.34212 & 6.43153 & 6.65156 & 6.29427 \\
\hline & $(0.14139)$ & $(0.13628)$ & $(0.06585)$ & $(0.11647)$ & $(0.11073)$ & $(0.05273)$ & $(0.12785)$ & $(0.12221)$ & $(0.05855)$ \\
\hline \multirow{2}{*}{70} & 6.31440 & 6.55586 & 6.16137 & 6.39833 & 6.59632 & 6.27610 & 6.36458 & 6.58232 & 6.22875 \\
\hline & $(0.10822)$ & $(0.10431)$ & $(0.04703)$ & $(0.08915)$ & $(0.08475)$ & $(0.04070)$ & $(0.09786)$ & $(0.09354)$ & $(0.03522)$ \\
\hline \multirow{2}{*}{100} & 6.13406 & 6.36862 & 6.09523 & 6.20961 & 6.40175 & 6.16118 & 6.14495 & 6.25773 & 6.11471 \\
\hline & $(0.06582)$ & $(0.06574)$ & $(0.03292)$ & $(0.05421)$ & $(0.05344)$ & $(0.02637)$ & $(0.05950)$ & $(0.05897)$ & $(0.02926)$ \\
\hline \multirow{2}{*}{150} & 6.08716 & 6.31992 & 6.04748 & 6.14353 & 6.33363 & 6.07259 & 6.08105 & 6.16165 & 6.02679 \\
\hline & $(0.04330)$ & $(0.04356)$ & $(0.02194)$ & $(0.03566)$ & $(0.03540)$ & $(0.01757)$ & $(0.03914)$ & $(0.03906)$ & $(0.01952)$ \\
\hline
\end{tabular}

\section{Conclusions and Recommendations}

The study has been carried out to investigate the performance of the Bayesian point and interval estimators of the parameters for Burr type VIII distribution based on five priors, three loss functions, and three censoring techniques. From the above analysis it can be concluded that amount of risk decreases and rate of convergence of estimates towards the true value increases with the increase in sample size. The performance of the inverse Levy prior has been the best among all priors under all cases. On the other hand, the quadratic loss function has been found to be the most efficient loss function under each prior and every censoring technique. Similarly, the estimates under singly type II censoring scheme are associated with the minimum amount of risks. So, on the whole, the use of inverse Levy prior along with 
TABLE 6: 95\% credible intervals under different priors and censoring techniques for $\Psi=2$.

\begin{tabular}{|c|c|c|c|c|c|c|c|c|c|}
\hline \multirow{2}{*}{$n$} & \multicolumn{3}{|c|}{ Left censored } & \multicolumn{3}{|c|}{ Singly type II censored } & \multicolumn{3}{|c|}{ Doubly type II censored } \\
\hline & LL & UL & $\mathrm{UL}-\mathrm{LL}$ & LL & UL & $\mathrm{UL}-\mathrm{LL}$ & LL & UL & $\mathrm{UL}-\mathrm{LL}$ \\
\hline & \multicolumn{9}{|c|}{ Uniform prior } \\
\hline 20 & 1.56781 & 2.75938 & 1.19157 & 1.73877 & 2.84372 & 1.10495 & 1.73962 & 2.90945 & 1.16983 \\
\hline 30 & 1.61913 & 2.59787 & 0.97874 & 1.79641 & 2.70600 & 0.90960 & 1.78866 & 2.72517 & 0.93650 \\
\hline 50 & 1.62627 & 2.53449 & 0.90822 & 1.80163 & 2.66082 & 0.85919 & 1.79136 & 2.66332 & 0.87196 \\
\hline 70 & 1.66166 & 2.45624 & 0.79458 & 1.83777 & 2.58974 & 0.75197 & 1.82279 & 2.59834 & 0.77556 \\
\hline 100 & 1.70699 & 2.32667 & 0.61968 & 1.85560 & 2.41714 & 0.56155 & 1.83423 & 2.40771 & 0.57348 \\
\hline \multirow[t]{2}{*}{150} & 1.75676 & 2.25937 & 0.50262 & 1.88471 & 2.34154 & 0.45683 & 1.85867 & 2.33087 & 0.47219 \\
\hline & \multicolumn{9}{|c|}{ Jeffreys prior } \\
\hline 20 & 1.56858 & 2.74542 & 1.17685 & 1.73708 & 2.80267 & 1.06559 & 1.73746 & 2.86972 & 1.13226 \\
\hline 30 & 1.61875 & 2.58540 & 0.96664 & 1.79389 & 2.66752 & 0.87362 & 1.78579 & 2.68849 & 0.90270 \\
\hline 50 & 1.62554 & 2.52254 & 0.89700 & 1.79889 & 2.62316 & 0.82427 & 1.78830 & 2.62765 & 0.83935 \\
\hline 70 & 1.66030 & 2.44505 & 0.78476 & 1.83458 & 2.55341 & 0.71884 & 1.79885 & 2.54065 & 0.74180 \\
\hline 100 & 1.70467 & 2.31670 & 0.61202 & 1.85182 & 2.38369 & 0.53187 & 1.79008 & 2.33228 & 0.54221 \\
\hline \multirow[t]{2}{*}{150} & 1.75374 & 2.25015 & 0.49640 & 1.88049 & 2.30946 & 0.42898 & 1.79945 & 2.24150 & 0.44204 \\
\hline & \multicolumn{9}{|c|}{ Exponential prior } \\
\hline 20 & 1.55362 & 2.71593 & 1.16230 & 1.71997 & 2.74490 & 1.02493 & 1.72025 & 2.78576 & 1.06552 \\
\hline 30 & 1.60306 & 2.55776 & 0.95470 & 1.77605 & 2.61266 & 0.83660 & 1.76796 & 2.61904 & 0.85109 \\
\hline 50 & 1.60971 & 2.49562 & 0.88591 & 1.78096 & 2.56925 & 0.78830 & 1.77039 & 2.64202 & 0.87163 \\
\hline 70 & 1.64399 & 2.41905 & 0.77506 & 1.81620 & 2.50101 & 0.68482 & 1.78077 & 2.49203 & 0.71126 \\
\hline 100 & 1.68773 & 2.29219 & 0.60446 & 1.83315 & 2.33487 & 0.50173 & 1.77199 & 2.31768 & 0.54570 \\
\hline \multirow[t]{2}{*}{150} & 1.73618 & 2.22645 & 0.49027 & 1.86144 & 2.26223 & 0.40079 & 1.78120 & 2.25684 & 0.47564 \\
\hline & \multicolumn{9}{|c|}{ Gamma prior } \\
\hline 20 & 1.62347 & 2.72707 & 1.10360 & 1.77884 & 2.78763 & 1.00880 & 1.77576 & 2.78477 & 1.00901 \\
\hline 30 & 1.66664 & 2.57312 & 0.90648 & 1.83130 & 2.65756 & 0.82626 & 1.82029 & 2.62195 & 0.80166 \\
\hline 50 & 1.67102 & 2.51219 & 0.84117 & 1.83474 & 2.61473 & 0.77998 & 1.82142 & 2.64617 & 0.82475 \\
\hline 70 & 1.70210 & 2.43802 & 0.73592 & 1.86815 & 2.54765 & 0.67951 & 1.82981 & 2.49792 & 0.66811 \\
\hline 100 & 1.74075 & 2.31468 & 0.57393 & 1.88157 & 2.38174 & 0.50017 & 1.81750 & 2.32602 & 0.50852 \\
\hline \multirow[t]{2}{*}{150} & 1.78615 & 2.25166 & 0.46551 & 1.90782 & 2.31004 & 0.40222 & 1.82464 & 2.26706 & 0.44242 \\
\hline & \multicolumn{9}{|c|}{ Inverse Levy prior } \\
\hline 20 & 1.60787 & 2.69784 & 1.08996 & 1.76125 & 2.73853 & 0.97728 & 1.75811 & 2.70183 & 0.94372 \\
\hline 30 & 1.65039 & 2.54567 & 0.89528 & 1.81304 & 2.61087 & 0.79783 & 1.80206 & 2.54396 & 0.74190 \\
\hline 50 & 1.65467 & 2.48544 & 0.83077 & 1.81640 & 2.56882 & 0.75242 & 1.80314 & 2.56750 & 0.76436 \\
\hline 70 & 1.68532 & 2.41214 & 0.72682 & 1.84939 & 2.50299 & 0.65360 & 1.81138 & 2.42371 & 0.61233 \\
\hline 100 & 1.72340 & 2.29024 & 0.56684 & 1.86256 & 2.34007 & 0.47751 & 1.79911 & 2.25700 & 0.45789 \\
\hline 150 & 1.77685 & 2.23660 & 0.45976 & 1.89728 & 2.27883 & 0.38156 & 1.81447 & 2.20874 & 0.39427 \\
\hline
\end{tabular}

quadratic loss function under singly type II censored samples can be preferred to estimate the parameter of Burr type VIII distribution.

\section{Appendix}

\section{Derivation of Different Expressions/Formulae}

The pdf of the Burr type VIII distribution given in (1) can be written as

$$
f(x, \psi) \propto \psi e^{-\psi\left\{(2 / \pi) \tan ^{-1}\left(e^{x}\right)\right\}^{-1}} .
$$

The CDF of the Burr type VIII distribution used in (2) can be written as

$$
F(x, \psi) \propto e^{-\psi\left\{(2 / \pi) \tan ^{-1}\left(e^{x}\right)\right\}^{-1}} .
$$

The likelihood function presented in (8) can be expressed as

$$
\begin{aligned}
L(\psi \mid x) \propto & \left\{e^{-\psi\left\{(2 / \pi) \tan ^{-1}\left(e^{x_{m+1}}\right)\right\}^{-1}}\right\}^{m} \\
& \times \prod_{i=m+1}^{n} \psi e^{-\psi\left\{(2 / \pi) \tan ^{-1}\left(e^{x_{i}}\right)\right\}^{-1}},
\end{aligned}
$$


TABLE 7: 95\% posterior predictive intervals under different priors and censoring techniques for $\Psi=2$.

\begin{tabular}{|c|c|c|c|c|c|c|c|c|c|}
\hline \multirow{2}{*}{$n$} & \multicolumn{3}{|c|}{ Left censored } & \multicolumn{3}{|c|}{ Singly type II censored } & \multicolumn{3}{|c|}{ Doubly type II censored } \\
\hline & LL & UL & $\mathrm{UL}-\mathrm{LL}$ & LL & UL & $\mathrm{UL}-\mathrm{LL}$ & LL & UL & $\mathrm{UL}-\mathrm{LL}$ \\
\hline & \multicolumn{9}{|c|}{ Uniform prior } \\
\hline 20 & 0.49523 & 13.47175 & 12.97652 & 0.54924 & 13.88351 & 13.33428 & 0.54950 & 14.20442 & 13.65491 \\
\hline 30 & 0.51144 & 12.68323 & 12.17179 & 0.56744 & 13.21114 & 12.64370 & 0.56500 & 13.30473 & 12.73974 \\
\hline 50 & 0.51370 & 12.37380 & 11.86010 & 0.56909 & 12.99057 & 12.42147 & 0.56585 & 13.00277 & 12.43692 \\
\hline 70 & 0.52488 & 11.99177 & 11.46689 & 0.58051 & 12.64354 & 12.06303 & 0.57578 & 12.68553 & 12.10975 \\
\hline 100 & 0.53920 & 11.35919 & 10.81999 & 0.58614 & 11.80088 & 11.21474 & 0.57939 & 11.75484 & 11.17545 \\
\hline \multirow[t]{2}{*}{150} & 0.55492 & 11.03062 & 10.47570 & 0.59534 & 11.43179 & 10.83645 & 0.58711 & 11.37969 & 10.79258 \\
\hline & \multicolumn{9}{|c|}{ Jeffreys prior } \\
\hline 20 & 0.49548 & 13.40360 & 12.90812 & 0.54870 & 13.68310 & 13.13440 & 0.54882 & 14.01045 & 13.46163 \\
\hline 30 & 0.51132 & 12.62235 & 12.11103 & 0.56665 & 13.02328 & 12.45663 & 0.56409 & 13.12565 & 12.56157 \\
\hline 50 & 0.51347 & 12.31546 & 11.80199 & 0.56823 & 12.80670 & 12.23848 & 0.56488 & 12.82862 & 12.26374 \\
\hline 70 & 0.52445 & 11.93714 & 11.41269 & 0.57950 & 12.46617 & 11.88667 & 0.56821 & 12.40388 & 11.83566 \\
\hline 100 & 0.53846 & 11.31051 & 10.77205 & 0.58495 & 11.63757 & 11.05262 & 0.56544 & 11.38658 & 10.82113 \\
\hline \multirow[t]{2}{*}{150} & 0.55396 & 10.98561 & 10.43164 & 0.59400 & 11.27517 & 10.68117 & 0.56840 & 10.94338 & 10.37497 \\
\hline & \multicolumn{9}{|c|}{ Exponential prior } \\
\hline 20 & 0.49075 & 13.25962 & 12.76887 & 0.54330 & 13.40106 & 12.85776 & 0.54339 & 13.60054 & 13.05716 \\
\hline 30 & 0.50637 & 12.48741 & 11.98104 & 0.56101 & 12.75544 & 12.19443 & 0.55846 & 12.78659 & 12.22813 \\
\hline 50 & 0.50847 & 12.18403 & 11.67556 & 0.56256 & 12.54351 & 11.98094 & 0.55922 & 12.89878 & 12.33956 \\
\hline 70 & 0.51930 & 11.81020 & 11.29091 & 0.57369 & 12.21035 & 11.63665 & 0.56250 & 12.16650 & 11.60400 \\
\hline 100 & 0.53311 & 11.19085 & 10.65774 & 0.57905 & 11.39922 & 10.82017 & 0.55973 & 11.31530 & 10.75557 \\
\hline \multirow[t]{2}{*}{150} & 0.54842 & 10.86990 & 10.32148 & 0.58798 & 11.04458 & 10.45660 & 0.56264 & 11.01827 & 10.45563 \\
\hline & \multicolumn{9}{|c|}{ Gamma prior } \\
\hline 20 & 0.51282 & 13.31401 & 12.80119 & 0.56189 & 13.60967 & 13.04778 & 0.56092 & 13.59571 & 13.03479 \\
\hline 30 & 0.52645 & 12.56240 & 12.03595 & 0.57846 & 12.97465 & 12.39619 & 0.57499 & 12.80080 & 12.22581 \\
\hline 50 & 0.52784 & 12.26493 & 11.73709 & 0.57955 & 12.76555 & 12.18600 & 0.57534 & 12.91904 & 12.34370 \\
\hline 70 & 0.53765 & 11.90282 & 11.36517 & 0.59010 & 12.43805 & 11.84795 & 0.57799 & 12.19526 & 11.61727 \\
\hline 100 & 0.54986 & 11.30065 & 10.75079 & 0.59434 & 11.62805 & 11.03371 & 0.57411 & 11.35602 & 10.78191 \\
\hline \multirow[t]{2}{*}{150} & 0.56420 & 10.99298 & 10.42878 & 0.60264 & 11.27800 & 10.67536 & 0.57636 & 11.06816 & 10.49180 \\
\hline & \multicolumn{9}{|c|}{ Inverse Levy prior } \\
\hline 20 & 0.50789 & 13.17130 & 12.66342 & 0.55634 & 13.36996 & 12.81362 & 0.55535 & 13.19078 & 12.63544 \\
\hline 30 & 0.52132 & 12.42838 & 11.90706 & 0.57270 & 12.74670 & 12.17400 & 0.56923 & 12.42004 & 11.85081 \\
\hline 50 & 0.52267 & 12.13433 & 11.61166 & 0.57376 & 12.54141 & 11.96765 & 0.56957 & 12.53496 & 11.96539 \\
\hline 70 & 0.53235 & 11.77647 & 11.24412 & 0.58418 & 12.22001 & 11.63583 & 0.57217 & 11.83295 & 11.26078 \\
\hline 100 & 0.54438 & 11.18133 & 10.63695 & 0.58834 & 11.42461 & 10.83627 & 0.56830 & 11.01905 & 10.45075 \\
\hline 150 & 0.56126 & 10.91945 & 10.35819 & 0.59931 & 11.12563 & 10.52632 & 0.57315 & 10.78344 & 10.21029 \\
\hline
\end{tabular}

$$
\begin{aligned}
& L(\psi \mid x) \propto \psi^{n-m} e^{-\psi\left[\sum_{i=m+1}^{n} \ln \left\{(2 / \pi) \tan ^{-1}\left(e^{x_{i}}\right)\right\}^{-1}\right]} \\
& \times e^{-\psi\left[m \ln \left\{(2 / \pi) \tan ^{-1}\left(e^{x_{m+1}}\right)\right\}^{-1}\right]}, \\
& L(\psi \mid x) \propto \psi^{n-m} e^{-\psi C_{1}(t)},
\end{aligned}
$$

where

$$
\begin{aligned}
C_{1}(x)= & \sum_{i=m+1}^{n} \ln \left\{\left(\frac{2}{\pi}\right) \tan ^{-1}\left(e^{x_{i}}\right)\right\}^{-1} \\
& +m \ln \left\{\left(\frac{2}{\pi}\right) \tan ^{-1}\left(e^{x_{m+1}}\right)\right\}^{-1} .
\end{aligned}
$$

The generalized (joint) version of priors presented in (3)-(7) can be written as

$$
p(\psi) \propto \psi^{-l_{1}+l_{3}(a-1)-0.5 l_{4}} e^{-\psi\left\{l_{2} c+l_{3} b+0.5 d l_{4}\right\}} .
$$

(A.3) Based on the density, kernel of the posterior distribution can be given as

$$
\begin{gathered}
p(\psi \mid x) \propto \psi^{n-m-l_{1}+l_{3}(a-1)-0.5 l_{4}} e^{-\psi\left\{C_{1}(x)+l_{2} c+l_{3} b+0.5 d l_{4}\right\}}, \\
p(\psi \mid x)=\frac{\psi^{n-m-l_{1}+l_{3}(a-1)-0.5 l_{4}} e^{-\psi\left\{C_{1}(x)+l_{2} c+l_{3} b+0.5 d l_{4}\right\}}}{\int_{0}^{\infty} \psi^{n-m-l_{1}+l_{3}(a-1)-0.5 l_{4}} e^{-\psi\left\{C_{1}(x)+l_{2} c+l_{3} b+0.5 d l_{4}\right\}} d \psi} .
\end{gathered}
$$


TABLE 8: Bayes estimates and posterior risks under real life data.

\begin{tabular}{|c|c|c|c|c|c|c|c|c|c|}
\hline \multirow{2}{*}{ Priors } & \multicolumn{3}{|c|}{ Left censored } & \multicolumn{3}{|c|}{ Singly type II censored } & \multicolumn{3}{|c|}{ Doubly type II censored } \\
\hline & LLF & PLF & QLF & LLF & PLF & QLF & LLF & PLF & QLF \\
\hline & \multicolumn{9}{|c|}{$10 \%$ censored } \\
\hline \multirow{2}{*}{ Uniform } & 47.24555 & 48.72147 & 46.64106 & 47.87348 & 49.02215 & 47.50956 & 47.62094 & 48.91817 & 47.15113 \\
\hline & $(3.30822)$ & $(2.49507)$ & $(0.01786)$ & $(2.86304)$ & $(2.28683)$ & $(0.01724)$ & $(3.01607)$ & $(2.43653)$ & $(0.01760)$ \\
\hline \multirow{2}{*}{ Jeffreys } & 46.66228 & 47.87151 & 46.01342 & 47.33033 & 48.21597 & 46.83223 & 47.03304 & 48.06477 & 46.51663 \\
\hline & $(3.26701)$ & $(2.48894)$ & $(0.01763)$ & $(2.82738)$ & $(2.28121)$ & $(0.01702)$ & $(2.97850)$ & $(2.43055)$ & $(0.01738)$ \\
\hline \multirow{2}{*}{ Exponential } & 46.08621 & 47.03637 & 45.39423 & 46.79335 & 47.42304 & 46.16456 & 46.45240 & 47.22627 & 45.89067 \\
\hline & $(3.21329)$ & $(2.42312)$ & $(0.01734)$ & $(2.78088)$ & $(2.22089)$ & $(0.01674)$ & $(2.92952)$ & $(2.36627)$ & $(0.01709)$ \\
\hline \multirow{2}{*}{ Gamma } & 46.40313 & 47.35982 & 45.70638 & 47.11513 & 47.74915 & 46.48201 & 46.77183 & 47.55102 & 46.20624 \\
\hline & $(2.93552)$ & $(2.33481)$ & $(0.01585)$ & $(2.54049)$ & $(2.13995)$ & $(0.01530)$ & $(2.67628)$ & $(2.28004)$ & $(0.01561)$ \\
\hline \multirow{3}{*}{ Inverse Levy } & 46.53661 & 47.52780 & 45.83237 & 47.25066 & 47.91850 & 46.61013 & 46.90637 & 47.71967 & 46.33360 \\
\hline & $(2.88725)$ & $(2.29642)$ & $(0.01558)$ & $(2.49872)$ & $(2.10476)$ & $(0.01505)$ & $(2.63227)$ & $(2.24255)$ & $(0.01536)$ \\
\hline & \multicolumn{9}{|c|}{$20 \%$ censored } \\
\hline \multirow{2}{*}{ Uniform } & 48.88463 & 50.33114 & 48.34057 & 49.75404 & 50.92134 & 49.35048 & 49.40203 & 50.34688 & 48.98630 \\
\hline & (3.40898) & $(2.57077)$ & $(0.01848)$ & $(2.94916)$ & $(2.37172)$ & $(0.01785)$ & $(3.12592)$ & $(2.50748)$ & $(0.01828)$ \\
\hline \multirow{2}{*}{ Jeffreys } & 47.86867 & 49.27538 & 47.14291 & 48.74232 & 49.75933 & 48.22909 & 48.59545 & 49.44202 & 48.29985 \\
\hline & $(3.34670)$ & $(2.56094)$ & $(0.01805)$ & $(2.90939)$ & $(2.35381)$ & $(0.01749)$ & $(3.04704)$ & $(2.49551)$ & $(0.01804)$ \\
\hline \multirow{2}{*}{ Exponential } & 47.13807 & 48.35455 & 46.45451 & 48.09022 & 48.68251 & 47.41832 & 47.50274 & 48.04644 & 47.55169 \\
\hline & $(3.28574)$ & $(2.48695)$ & $(0.01766)$ & $(2.85620)$ & $(2.27935)$ & $(0.01708)$ & $(2.99525)$ & $(2.40593)$ & $(0.01771)$ \\
\hline \multirow{2}{*}{ Gamma } & 47.42154 & 48.43907 & 46.48440 & 48.00535 & 48.93807 & 47.30361 & 47.70787 & 48.24894 & 47.67193 \\
\hline & (2.99705) & $(2.38132)$ & $(0.01610)$ & $(2.58490)$ & $(2.19134)$ & $(0.01556)$ & $(2.72857)$ & $(2.30718)$ & $(0.01590)$ \\
\hline \multirow{2}{*}{ Inverse Levy } & 47.22389 & 48.27313 & 46.52757 & 47.91474 & 48.90824 & 47.34369 & 47.79353 & 48.24738 & 46.99236 \\
\hline & $(2.91876)$ & $(2.32932)$ & $(0.01579)$ & $(2.52577)$ & $(2.13919)$ & $(0.01522)$ & $(2.66669)$ & $(2.26701)$ & $(0.01553)$ \\
\hline
\end{tabular}

TABLE 9: 95\% credible intervals under real life data.

\begin{tabular}{|c|c|c|c|c|c|c|c|c|c|}
\hline \multirow{2}{*}{ Priors } & \multicolumn{3}{|c|}{ Left censored } & \multicolumn{3}{|c|}{ Singly type II censored } & \multicolumn{3}{|c|}{ Doubly type II censored } \\
\hline & LL & $\mathrm{UL}$ & $\mathrm{UL}-\mathrm{LL}$ & LL & $\mathrm{UL}$ & $\mathrm{UL}-\mathrm{LL}$ & LL & $\mathrm{UL}$ & $\mathrm{UL}-\mathrm{LL}$ \\
\hline & \multicolumn{9}{|c|}{$10 \%$ censored } \\
\hline Uniform & 43.68060 & 50.81050 & 7.12990 & 44.55706 & 51.18990 & 6.63284 & 44.21704 & 51.02484 & 6.80780 \\
\hline Jeffreys & 43.11961 & 50.20496 & 7.08535 & 44.03463 & 50.62604 & 6.59140 & 43.65041 & 50.41568 & 6.76526 \\
\hline Exponential & 42.57279 & 49.59964 & 7.02685 & 43.52486 & 50.06184 & 6.53698 & 43.09770 & 49.80711 & 6.70941 \\
\hline Gamma & 43.04499 & 49.76127 & 6.71627 & 43.99110 & 50.23916 & 6.24806 & 43.56540 & 49.97826 & 6.41286 \\
\hline \multirow[t]{2}{*}{ Inverse Levy } & 43.20620 & 49.86702 & 6.66082 & 44.15242 & 50.34889 & 6.19647 & 43.72641 & 50.08633 & 6.35992 \\
\hline & \multicolumn{9}{|c|}{$20 \%$ censored } \\
\hline Uniform & 42.72716 & 52.83739 & 10.11023 & 43.22736 & 52.78174 & 9.55438 & 43.26458 & 52.76883 & 9.50425 \\
\hline Jeffreys & 42.25671 & 52.09809 & 9.84138 & 42.72587 & 52.09732 & 9.37145 & 42.83694 & 51.57464 & 8.73770 \\
\hline Exponential & 41.89867 & 51.40512 & 9.50645 & 42.34254 & 51.34485 & 9.00231 & 42.41062 & 50.82486 & 8.41424 \\
\hline Gamma & 42.50686 & 51.06047 & 8.55360 & 42.91141 & 51.31689 & 8.40548 & 42.89212 & 50.76187 & 7.86975 \\
\hline Inverse Levy & 42.74227 & 50.65512 & 7.91285 & 43.63056 & 51.40093 & 7.77038 & 43.07501 & 50.82409 & 7.74908 \\
\hline
\end{tabular}

The complete version of the posterior distribution can be presented as

$$
\begin{aligned}
& p(\psi \mid x) \\
& =\frac{\left\{C_{1}(x)+l_{2} c+l_{3} b+0.5 d l_{4}\right\}^{n-m+1-l_{1}+l_{3}(a-1)-0.5 l_{4}}}{\Gamma\left(n-m+1-l_{1}+l_{3}(a-1)-0.5 l_{4}\right)}
\end{aligned}
$$

$$
\begin{aligned}
& \times \psi^{n-m-l_{1}+l_{3}(a-1)-0.5 l_{4}} \\
& \times e^{-\psi\left\{C_{1}(x)+l_{2} c+l_{3} b+0.5 d l_{4}\right\}} .
\end{aligned}
$$

The Bayes estimator under the LLF can be derived by using the following formula:

$$
\psi_{\mathrm{LLF}}=-\ln \left[E\left(e^{-\psi}\right)\right]
$$


TABLE 10: 95\% posterior predictive intervals under real life data.

\begin{tabular}{|c|c|c|c|c|c|c|c|c|c|}
\hline \multirow{2}{*}{ Priors } & \multicolumn{3}{|c|}{ Left censored } & \multicolumn{3}{|c|}{ Singly type II censored } & \multicolumn{3}{|c|}{ Doubly type II censored } \\
\hline & LL & $\mathrm{UL}$ & $\mathrm{UL}$ - LL & LL & UL & $\mathrm{UL}-\mathrm{LL}$ & LL & UL & $\mathrm{UL}-\mathrm{LL}$ \\
\hline & \multicolumn{9}{|c|}{$10 \%$ censored } \\
\hline Uniform & 13.79766 & 248.06530 & 234.26764 & 14.07452 & 249.91759 & 235.84308 & 13.96711 & 249.11174 & 235.14463 \\
\hline Jeffreys & 13.62046 & 245.10895 & 231.48849 & 13.90949 & 247.16474 & 233.25524 & 13.78813 & 246.13772 & 232.34960 \\
\hline Exponential & 13.44773 & 242.15368 & 228.70595 & 13.74847 & 244.41022 & 230.66175 & 13.61354 & 243.16658 & 229.55304 \\
\hline Gamma & 13.59689 & 242.94278 & 229.34589 & 13.89574 & 245.27592 & 231.38018 & 13.76128 & 244.00217 & 230.24089 \\
\hline \multirow[t]{2}{*}{ Inverse Levy } & 13.64781 & 243.45907 & 229.81126 & 13.94670 & 245.81164 & 231.86494 & 13.81213 & 244.52978 & 230.71765 \\
\hline & \multicolumn{9}{|c|}{$20 \%$ censored } \\
\hline Uniform & 13.49650 & 257.96091 & 244.46442 & 13.65450 & 257.68922 & 244.03472 & 13.66625 & 257.62619 & 243.95994 \\
\hline Jeffreys & 13.34789 & 254.35153 & 241.00364 & 13.49609 & 254.34777 & 240.85168 & 13.53117 & 251.79596 & 238.26479 \\
\hline Exponential & 13.23479 & 250.96833 & 237.73354 & 13.37500 & 250.67409 & 237.29908 & 13.39651 & 248.13541 & 234.73890 \\
\hline Gamma & 13.42691 & 249.28570 & 235.85879 & 13.55470 & 250.53758 & 236.98288 & 13.54860 & 247.82788 & 234.27928 \\
\hline Inverse Levy & 13.50127 & 247.30671 & 233.80544 & 13.78186 & 250.94788 & 237.16602 & 13.60637 & 248.13165 & 234.52528 \\
\hline
\end{tabular}

where

$$
\begin{aligned}
& E\left(e^{-\psi}\right)= \frac{\left\{C_{1}(t)+l_{2} c+l_{3} b+0.5 d l_{4}\right\}^{n-m+1-l_{1}+l_{3}(a-1)-0.5 l_{4}}}{\Gamma\left(n-m+1-l_{1}+l_{3}(a-1)-0.5 l_{4}\right)} \\
& \times \int_{0}^{\infty} \psi^{n-m-l_{1}+l_{3}(a-1)-0.5 l_{4}} \\
& \times e^{-\psi\left\{C_{1}(x)+l_{2} c+l_{3} b+0.5 d l_{4}+1\right\}} d \psi, \\
& E\left(e^{-\psi}\right) \\
&=\left\{\left(C_{1}(x)+l_{2} c+l_{3} b+0.5 d l_{4}\right)\right. \\
& \quad \times\left(C_{1}(x)+l_{2} c+l_{3} b+0.5 d l_{4}\right. \\
&\left.\quad+1)^{-1}\right\}^{n-m+1-l_{1}+l_{3}(a-1)-0.5 l_{4}} .
\end{aligned}
$$

Therefore,

$$
\begin{aligned}
\psi_{\mathrm{LLF}}= & -\ln \left[E\left(e^{-\psi}\right)\right] \\
= & -\left\{n-m+1-l_{1}+l_{3}(a-1)-0.5 l_{4}\right\} \\
& \times \ln \left\{\frac{C_{1}(x)+l_{2} c+l_{3} b+0.5 d l_{4}}{C_{1}(x)+l_{2} c+l_{3} b+0.5 d l_{4}+1}\right\} .
\end{aligned}
$$

The formula for the derivation of posterior risk under LLF is

$$
\rho\left(\psi_{\mathrm{LLF}}\right)=E(\psi)-\psi_{\mathrm{LLF}}
$$

where

$$
\begin{aligned}
E(\psi)= & \frac{\left\{C_{1}(x)+l_{2} c+l_{3} b+0.5 d l_{4}\right\}^{n-m+1-l_{1}+l_{3}(a-1)-0.5 l_{4}}}{\Gamma\left(n-m+1-l_{1}+l_{3}(a-1)-0.5 l_{4}\right)} \\
& \times \int_{0}^{\infty} \psi^{n-m+1-l_{1}+l_{3}(a-1)-0.5 l_{4}} \\
& \times e^{-\psi\left\{C_{1}(x)+l_{2} c+l_{3} b+0.5 d l_{4}\right\}} d \psi \\
E &
\end{aligned}
$$

Therefore, the posterior risk under LLF can be given as

$$
\begin{aligned}
\rho\left(\psi_{\mathrm{LLF}}\right)= & \frac{n-m+1-l_{1}+l_{3}(a-1)-0.5 l_{4}}{C_{1}(x)+l_{2} c+l_{3} b+0.5 d l_{4}} \\
& +\left\{n-m+1-l_{1}+l_{3}(a-1)-0.5 l_{4}\right\} \\
& \times \ln \left\{\frac{C_{1}(x)+l_{2} c+l_{3} b+0.5 d l_{4}}{C_{1}(x)+l_{2} c+l_{3} b+0.5 d l_{4}+1}\right\} .
\end{aligned}
$$

The formula for the derivation of the Bayes estimator under PLF is

$$
\psi_{\mathrm{PLF}}=\left[E\left(\psi^{2}\right)\right]^{1 / 2}
$$


where

$$
\begin{aligned}
& E\left(\psi^{2}\right) \\
& =\frac{\left\{C_{1}(x)+l_{2} c+l_{3} b+0.5 d l_{4}\right\}^{n-m+1-l_{1}+l_{3}(a-1)-0.5 l_{4}}}{\Gamma\left(n-m+1-l_{1}+l_{3}(a-1)-0.5 l_{4}\right)} \\
& \times \int_{0}^{\infty} \psi^{n-m+2-l_{1}+l_{3}(a-1)-0.5 l_{4}} \\
& \quad \times e^{-\psi\left\{C_{1}(x)+l_{2} c+l_{3} b+0.5 d l_{4}\right\}} d \psi \\
& E\left(\psi^{2}\right)=\left\{n-m+1-l_{1}+l_{3}(a-1)-0.5 l_{4}\right\} \\
& \quad \times\left\{n-m+2-l_{1}+l_{3}(a-1)-0.5 l_{4}\right\} \\
& \quad \times\left(\left\{C_{1}(x)+l_{2} c+l_{3} b+0.5 d l_{4}\right\}^{2}\right)^{-1} .
\end{aligned}
$$

Therefore,

$$
\begin{aligned}
\psi_{\mathrm{PLF}}= & {\left[E\left(\psi^{2}\right)\right]^{1 / 2} } \\
= & \left(\left\{n-m+1-l_{1}+l_{3}(a-1)-0.5 l_{4}\right\}\right. \\
& \left.\times\left\{n-m+2-l_{1}+l_{3}(a-1)-0.5 l_{4}\right\}\right)^{1 / 2} \\
& \times\left(C_{1}(x)+l_{2} c+l_{3} b+0.5 d l_{4}\right)^{-1} .
\end{aligned}
$$

The formula for the derivation of posterior risk under PLF is

$$
\psi_{\mathrm{PLF}}=2\left(\psi_{\mathrm{PLF}}-E(\psi)\right)
$$

Therefore, the posterior risk under PLF is

$$
\begin{aligned}
\rho\left(\psi_{\mathrm{PLF}}\right)=2[( & \left\{n-m+1-l_{1}+l_{3}(a-1)-0.5 l_{4}\right\} \\
& \left.\times\left\{n-m+2-l_{1}+l_{3}(a-1)-0.5 l_{4}\right\}\right)^{1 / 2} \\
& \times\left(C_{1}(x)+l_{2} c+l_{3} b+0.5 d l_{4}\right)^{-1} \\
& -\left\{n-m+1-l_{1}+l_{3}(a-1)-0.5 l_{4}\right\} \\
& \left.\times\left(C_{1}(x)+l_{2} c+l_{3} b+0.5 d l_{4}\right)^{-1}\right] .
\end{aligned}
$$

The Bayes estimator under QLF can be derived by using the following formula:

$$
\psi_{\mathrm{QLF}}=E\left(\psi^{-1}\right)\left\{E\left(\psi^{-2}\right)\right\}^{-1}
$$

where

$$
\begin{aligned}
& E\left(\psi^{-1}\right) \\
& =\frac{\left\{C_{1}(x)+l_{2} c+l_{3} b+0.5 d l_{4}\right\}^{n-m+1-l_{1}+l_{3}(a-1)-0.5 l_{4}}}{\Gamma\left(n-m+1-l_{1}+l_{3}(a-1)-0.5 l_{4}\right)} \\
& \times \int_{0}^{\infty} \psi^{n-m-1-l_{1}+l_{3}(a-1)-0.5 l_{4}} \\
& \times e^{-\psi\left\{C_{1}(x)+l_{2} c+l_{3} b+0.5 d l_{4}\right\}} d \psi, \\
& E\left(\psi^{-1}\right)=\frac{C_{1}(x)+l_{2} c+l_{3} b+0.5 d l_{4}}{n-m-l_{1}+l_{3}(a-1)-0.5 l_{4}}, \\
& E\left(\psi^{-2}\right) \\
& =\frac{\left\{C_{1}(x)+l_{2} c+l_{3} b+0.5 d l_{4}\right\}^{n-m+1-l_{1}+l_{3}(a-1)-0.5 l_{4}}}{\Gamma\left(n-m+1-l_{1}+l_{3}(a-1)-0.5 l_{4}\right)} \\
& \times \int_{0}^{\infty} \psi^{n-m-2-l_{1}+l_{3}(a-1)-0.5 l_{4}} \\
& \times e^{-\psi\left\{C_{1}(x)+l_{2} c+l_{3} b+0.5 d l_{4}\right\}} d \psi, \\
& \mathrm{E}\left(\psi^{-2}\right)=\left\{C_{1}(x)+l_{2} c+l_{3} b+0.5 d l_{4}\right\}^{2} \\
& \times\left(\left\{n-m-l_{1}+l_{3}(a-1)-0.5 l_{4}\right\}\right. \\
& \left.\times\left\{n-m-1-l_{1}+l_{3}(a-1)-0.5 l_{4}\right\}\right)^{-1} .
\end{aligned}
$$

Hence, the Bayes estimator under the QLF is

$$
\begin{aligned}
\psi_{\mathrm{QLF}} & =E\left(\psi^{-1}\right)\left\{E\left(\psi^{-2}\right)\right\}^{-1} \\
& =\frac{C_{1}(x)+l_{2} c+l_{3} b+0.5 d l_{4}}{n-m-1-l_{1}+l_{3}(a-1)-0.5 l_{4}} .
\end{aligned}
$$

Similarly, the expression for the posterior risk under QLF is

$$
\rho\left(\psi_{\mathrm{QLF}}\right)=1-\frac{\left\{E\left(\psi^{-1}\right)\right\}^{2}}{E\left(\psi^{-2}\right)}=\frac{1}{n-m-l_{1}+l_{3}(a-1)-0.5 l_{4}} .
$$

The derivation of Bayes estimators and posterior risks under other censoring schemes can be obtained in a similar manner.

\section{Conflict of Interests}

The author declares that there is no conflict of interests regarding the publication of this paper.

\section{References}

[1] I. W. Burr, "Cumulative frequency functions," Annals of Mathematical Statistics, vol. 13, pp. 215-232, 1942.

[2] A. A. Soliman, "Reliability estimation in a generalized lifemodel with application to the Burr-XII," IEEE Transactions on Reliability, vol. 51, no. 3, pp. 337-343, 2002. 
[3] N. K. Joshi, "Bayesian estimation of reliability of generalized Burr life time distribution under precautionary loss function with censoring," Journal of International Academy of Physical Sciences, vol. 8, pp. 69-75, 2004.

[4] A. Asgharzadeh and R. Valiollahi, "Estimation based on progressively censored data from the Burr model," International Mathematical Forum, vol. 3, no. 41-44, pp. 2113-2121, 2008.

[5] M. Saleem and M. Aslam, "On Bayesian analysis of the Rayleigh survival time assuming the random censor time," Pakistan Journal of Statistics, vol. 25, no. 2, pp. 71-82, 2009.

[6] E. K. AL-Hussaini and M. Hussein, "Estimation using censored data from exponentiated Burr type XII population," Open Journal of Statistics, vol. 1, no. 2, pp. 33-45, 2011.

[7] A. A. Soliman, A. H. Ellah, N. A. AAbou-Elheggag et al., "Bayesian inference and prediction of Burr type XII distribution for progressive first failure censored sampling," Intelligent Information Management, vol. 3, pp. 175-185, 2011.

[8] Y. Singh, "Unbiased estimation in Burr distribution," Journal of Mathematical and Computational Science, vol. 2, no. 4, pp. 830835, 2012.

[9] N. Feroze and M. Aslam, "Bayesian analysis of Gumbel type II distribution under doubly censored samples using different loss functions," Caspian Journal of Applied Sciences Research, vol. 1, pp. 1-10, 2012.

[10] P. S. Laplace, Theorie Analytique des Probabilities, Veuve Courcier, Paris, France, 1812.

[11] H. Jeffreys, Theory of Probability, Clarendon Press, Oxford, UK, 3rd edition, 1961.

[12] L. B. Klebanov, “"Universal” loss functions and unbiased estimates," Doklady Akademii Nauk SSSR, vol. 203, pp. 1249-1251, 1972.

[13] J. G. Norström, "The use of precautionary loss functions in risk analysis," IEEE Transactions on Reliability, vol. 45, no. 3, pp. 400-403, 1996.

[14] F. J. Samaniego, A Comparison of the Bayesian and Frequentist Approaches to Estimation, Springer Series in Statistics, Springer, New York, NY, USA, 2010.

[15] W. M. Bolstad, Introduction to Bayesian Statistics, John Wiley \& Sons, Hoboken, NJ, USA, 2004.

[16] P. Congdon, Bayesian Statistical Modelling, Wiley Series in Probability and Statistics, John Wiley \& Sons, Chichester, UK, 2nd edition, 2006.

[17] A. K. Bansal, Bayesian Parametric Inference, Narosa Publishing, New Delhi, India, 2007.

[18] J. F. Lawless, Statistical Models and Methods for Lifetime Data, Wiley Series in Probability and Statistics, John Wiley \& Sons, Hoboken, NJ, USA, 2nd edition, 2003. 


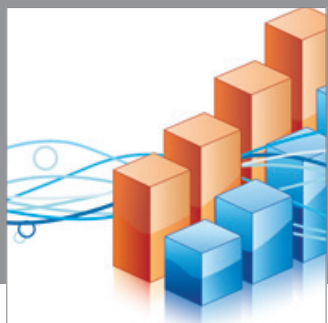

Advances in

Operations Research

mansans

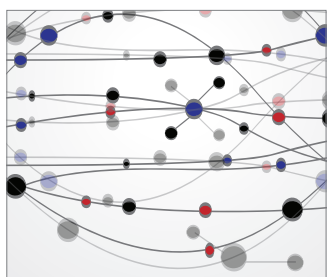

The Scientific World Journal
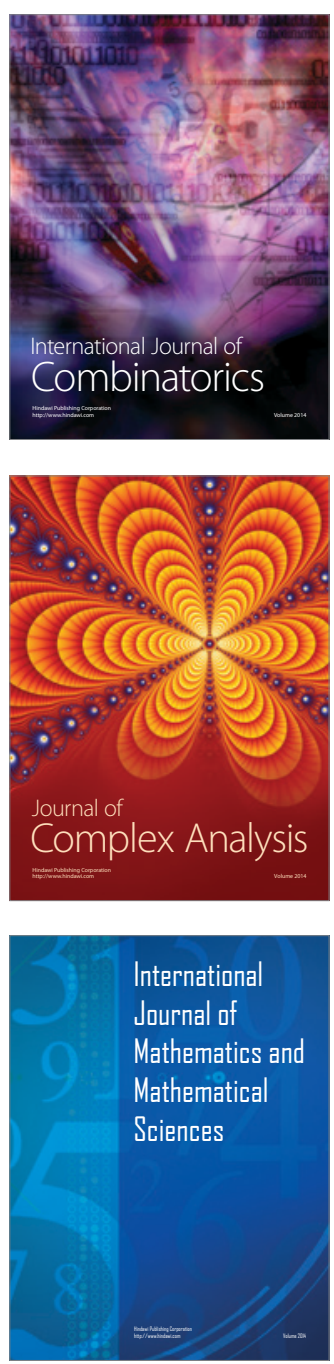
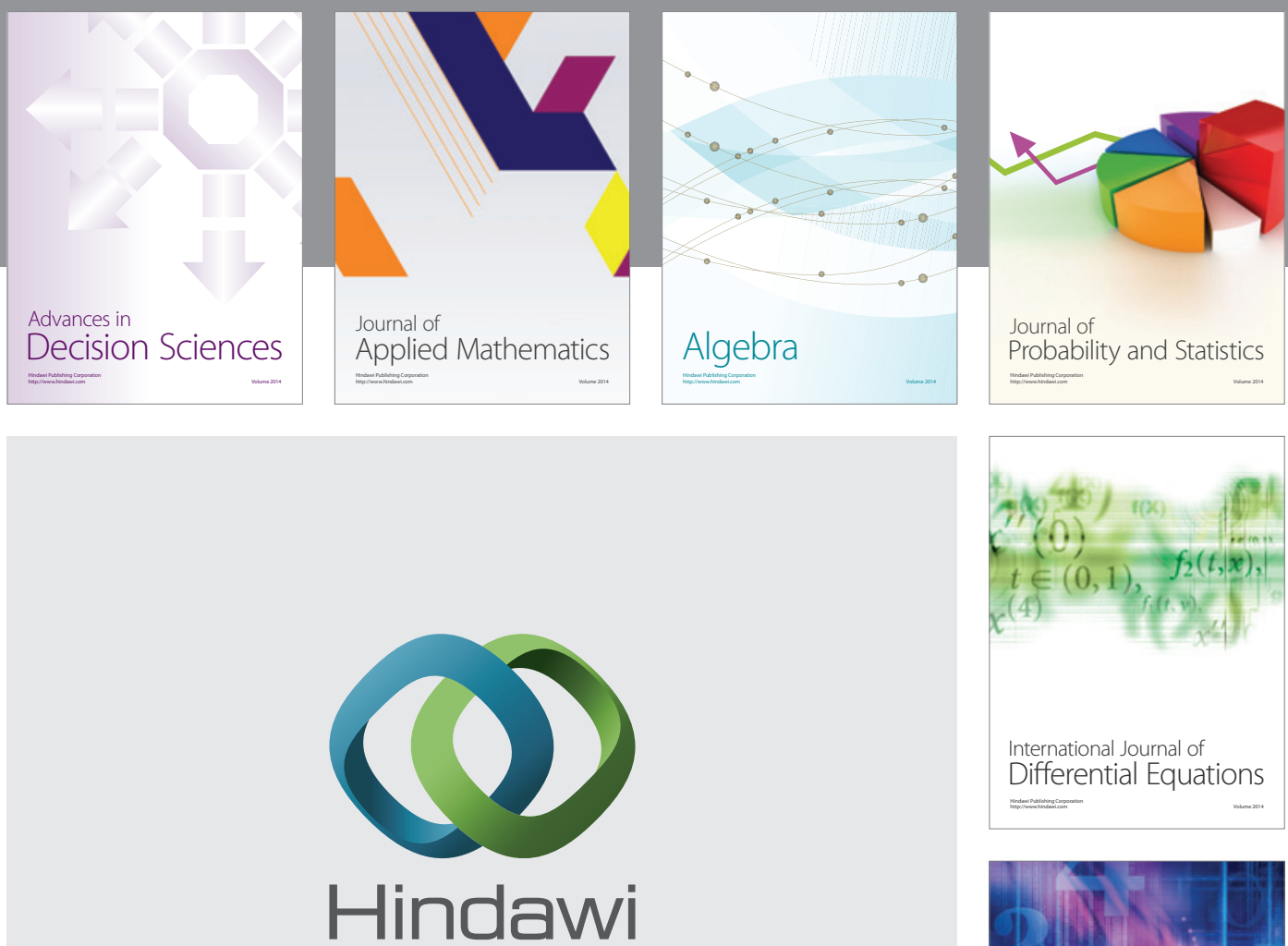

Submit your manuscripts at http://www.hindawi.com
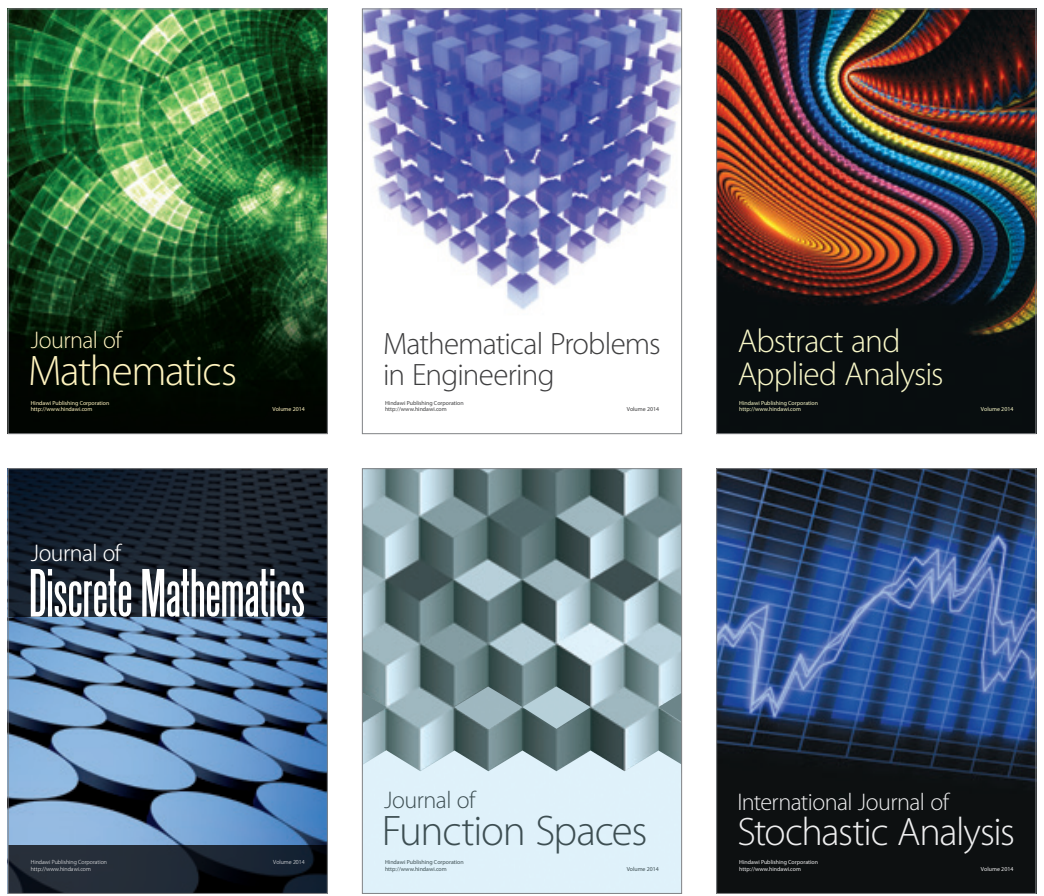

Journal of

Function Spaces

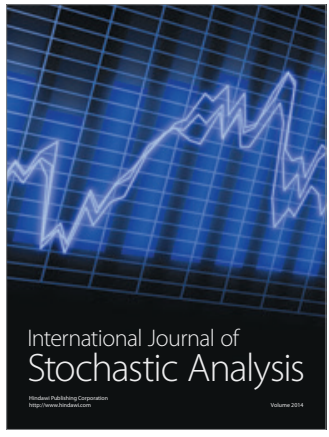

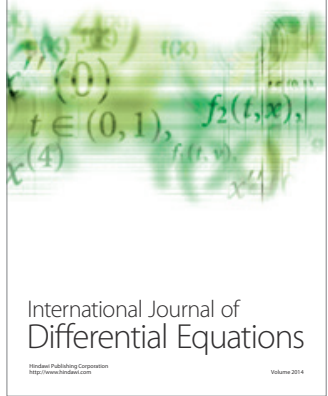
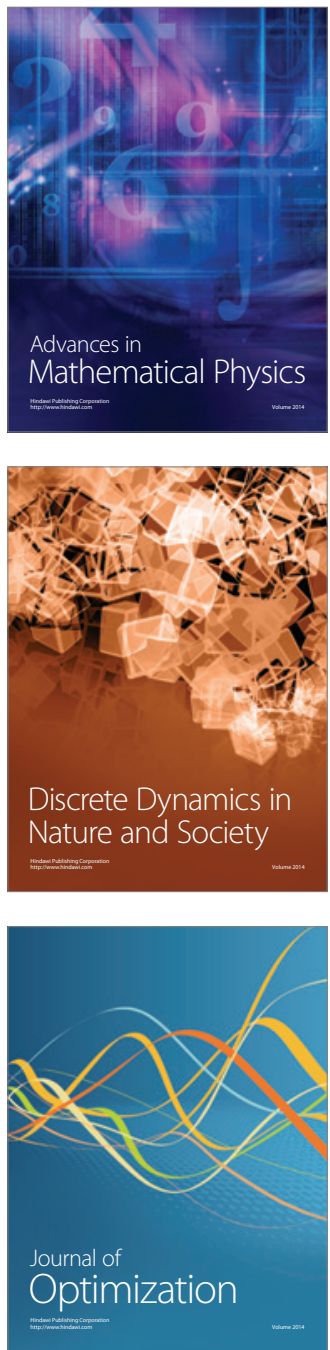